\title{
MICROSPECTRAL ANALYSIS OF QUASINILPOTENT OPERATORS
}

\author{
JARMO MALINEN, OLAVI NEVANLINNA, AND JAROSLAV ZEMÁNEK
}

\begin{abstract}
We develop a microspectral theory for quasinilpotent linear operators $Q$ (i.e., those with $\sigma(Q)=\{0\}$ ) in a Banach space. For such operators, the classical spectral theory gives little information. Deeper structure can be obtaind from microspectral sets in $\mathbb{C}$ as defined below. Such sets describe, e.g., semigroup generation, various resolvent properties, power boundedness as well as Tauberian properties associated to $z Q$ for $z \in \mathbb{C}$.
\end{abstract}

\section{INTRODUCTION}

Let $T$ be a bounded linear operator on a complex Banach space $X$ with its spectrum denoted by $\sigma(T)$. Local spectral theory deals with the local resolvent

$$
\lambda \mapsto(\lambda-T)^{-1} x, \quad x \in X .
$$

The domain and the analytic properties of such functions depend on the choice of the vector $x$; see [NRR87, FNRR90] for background in this area. In this paper we discuss related microspectral questions.

Let $\lambda_{0} \in \sigma(T)$ be an isolated point. If $\lambda_{0}$ is a pole of the global resolvent

$$
\lambda \mapsto(\lambda-T)^{-1}, \quad \lambda \notin \sigma(T),
$$

the the resolvent appears of the same size when approaching the singularity $\lambda_{0}$ from all directions; see Proposition 2.2 below. If, however, $\lambda_{0}$ is an essential singularity, then the growth depends on the direction from which the singularity is approached. In order to study this in a more detailed manner, we proceed as follows:

Let $P_{0}$ be the Riesz spectral projection of $X$ to the invariant subspace with respect to $\lambda_{0} \in \sigma(T)$, and define $X_{0}:=P X$ and $T_{0}=T \mid X_{0}$. Then clearly $Q:=$ $T_{0}-\lambda_{0}$ is a quasinilpotent operator on $X_{0}$. Rather than studying $(\lambda-Q)^{-1}$ for $\lambda \neq 0$, we change the variable and consider the entire function

$$
z \mapsto(I-z Q)^{-1}, \quad z \in \mathbb{C} .
$$

Refined spectral information for $Q$ can be obtained from the mapping

$$
j \mapsto(I+z Q)^{j}, \quad j \in \mathbb{N}:=\{1,2, \ldots\}
$$

in terms of the set

$$
\mathcal{B}_{Q}:=\left\{z \in \mathbb{C}: \exists N_{z}<\infty \text { such that }\left\|(1+z Q)^{j}\right\| \leq N_{z} \text { for all } j \in \mathbb{N}\right\} ;
$$

i.e., our requirement is the power-boundedness at the point $z$ with the bound $N_{z}$ that possibly depends on $z \in \mathcal{B}_{Q}$. It is easy to see that always $0 \in \mathcal{B}_{Q}$ and that $\mathcal{B}_{Q}$ is convex by the binomial formula for commuting operators.

1991 Mathematics Subject Classification. 47A10, 47B06, 47B10, 47B60.

Key words and phrases. Quasinilpotent, power-bounded, semigroup, microspectral. 
In addition to the power-bounded set $\mathcal{B}_{Q}$, a number of additional sets in $\mathbb{C}$ are defined in $(2.1)-(2.6)$ below. Studying these sets is a powerful tool for understanding the asymptotic behaviour of powers of the families of operators

$$
T(z):=I+z Q \quad \text { and } \quad T_{z}:=(I-z Q)^{-1}
$$

for complex $z$. Intuitively speaking, the proposed microspectal analysis amounts to looking at $Q$ from all possible directions and using all possible magnifying glasses.

We shall see below in Section 3 that topological properties of a point $z_{0}$ with respect to the set $\mathcal{B}_{Q}$ ( such as $z_{0} \in \mathcal{B}_{Q}^{\circ}$, the open interior of $\mathcal{B}_{Q}$ ) correspond to additional properties of powers $T(z)^{j}$ (such as growth condition on their consecutive differences $\left.(I-T(z)) T(z)^{j}\right)$ or the analytic properties of the resolvent (1.1) (like the Ritt resolvent condition).

In particular, it is known that the differences of consecutive powers $(I-T(z)) T(z)^{j}$ cannot decay arbitrarily fast since either $\liminf _{j \rightarrow \infty}(j+1)\left\|(I-T(z)) T(z)^{j}\right\| \geq$ $1 / e$ or $Q=0$; see [Est83, Ber83, KMSOT04, MNTY07]. The question arises whether $z \in \mathcal{B}_{Q}$ if the fastest possible speed of decay is attained, i.e.,

$$
\sup _{j \geq 1}(j+1)\left\|(I-T(z)) T(z)^{j}\right\|<\infty .
$$

Theorem 6.3 gives an affirmative answer for real operators

Notation. The bounded linear operators in a Banach space $X$ are denoted by $\mathcal{L}(X)$. The norm of $X$ and induced operator norm of $\mathcal{L}(X)$ are both denoted by $\|\cdot\|$. Throughout this paper we assume that $Q \in \mathcal{L}(X)$ with $\sigma(Q)=\{0\}$.

The natural numbers are $\mathbb{N}:=\{1,2, \ldots\}$. The complex plane and the real axis are denoted by $\mathbb{C}$ and $\mathbb{R}$, respectively. For any set $A \subset \mathbb{C}$, we denote by $\bar{A}, A^{c}$, $\partial A$, and $A^{\circ}$ the closure, complement, boundary, and the (open) interior of $A$, respectively. The positive real axis is denoted by $\mathbb{R}_{+}=(0, \infty)$ with $\overline{\mathbb{R}}_{+}=[0, \infty)$, and $\mathbb{D}_{z_{0}, r}:=\left\{z \in \mathbb{C}:\left|z-x_{0}\right|<r\right\}$. We define the unit disc $\mathbb{D}:=\mathbb{D}_{0,1}$ and its boundary, the unit circle $\mathbb{T}:=\partial \mathbb{D}$. If $A, B \subset \mathbb{C}$, we define their product set by $A B:=\{z s: z \in A$ and $s \in B\}$. We say that a set $A \subset \mathbb{C}$ is star-like or that it consists of full rays if

$$
A(0,1] \subset A \quad \text { or } \quad A \mathbb{R}_{+} \subset A,
$$

respectively. Note that the set $\{0\}$ satisfies both of these conditions.

Sectors are convex sets that consist of full rays. We denote the balanced open sectors in $\mathbb{C}$ by

$$
\Sigma_{\theta}:=\left\{r e^{i \theta_{0}}: r>0 \text { and } \theta_{0} \in(-\theta, \theta)\right\} \quad \text { for } \quad 0<\theta<\pi .
$$

We write $\mathbb{C}_{+}:=\Sigma_{\pi / 2}$. General open sectors are the sets $e^{i \phi} \Sigma_{\theta}$ for $\phi \in[-\pi, \pi)$ and $\theta \in(0, \pi)$. The central angle of $e^{i \phi} \Sigma_{\theta}$ is defined as $2 \theta$. Closed sectors are closures of open sectors or rays $e^{i \phi} \overline{\mathbb{R}}_{+}$for some $\phi \in[-\pi, \pi)$. 


\section{ELEMENTARY PROPERTIES}

In addition to the set $\mathcal{B}_{Q}$ already introduced in (1.2), we also consider the sets

$$
\begin{aligned}
\mathcal{A}_{Q}^{k} & :=\left\{z \in \mathbb{C}: \limsup _{s \rightarrow+\infty}\left\|(1-s z Q)^{-k}\right\|<\infty\right\} \quad \text { for } \quad k \in \mathbb{N}, \\
\mathcal{G}_{Q} & :=\left\{z \in \mathbb{C}: \limsup _{t \rightarrow+\infty}\left\|e^{t z Q}\right\|<\infty\right\}, \\
\mathcal{K}_{Q} & :=\left\{z \in \mathbb{C}: \sup _{\operatorname{Re} s>-1 / 2}(|s+1|-|s|)\left\|(1-s z Q)^{-1}\right\|<\infty\right\}, \\
\mathcal{K}_{Q}^{\infty} & :=\left\{z \in \mathbb{C}: \sup _{\operatorname{Re} s>-1 / 2, k \in \mathbb{N}}(|s+1|-|s|)^{k}\left\|(1-s z Q)^{-k}\right\|<\infty\right\}, \\
\mathcal{R}_{Q} & :=\left\{z \in \mathbb{C}: \sup _{\operatorname{Re} s>-1 / 2}\left\|(1-s z Q)^{-1}\right\|<\infty\right\}, \quad \text { and } \\
\mathcal{T}_{Q}^{\alpha} & :=\left\{z \in \mathbb{C}: \limsup _{j \rightarrow \infty}(j+1)^{\alpha}\left\|z Q(1+z Q)^{j}\right\|<\infty\right\} \quad \text { for } \quad \alpha>0
\end{aligned}
$$

as well as the constants defined by

$$
\begin{aligned}
& M_{z}:=\sup _{j \geq 0}(j+1)\left\|z Q(1+z Q)^{j}\right\| \quad \text { for } \quad z \in \mathcal{T}_{Q}, \quad \text { and } \\
& N_{z}:=\sup _{j \geq 0}\left\|(1+z Q)^{j}\right\| \quad \text { for } \quad z \in \mathcal{B}_{Q} .
\end{aligned}
$$

We shall abbreviate $\mathcal{T}_{Q}^{1}=\mathcal{T}_{Q}$ and $\mathcal{A}_{Q}^{1}=\mathcal{A}_{Q}$. If $Q=0$, then all these sets coincide with $\mathbb{C}$.

The set $\mathcal{A}_{Q}^{k}$ is referred to as the Abel set of order $k$ for obvious reasons. The Kreiss set is so defined that $z \in \mathcal{K}_{Q}$ if and only if $T(z)$ in (1.3) satisfies the Kreiss resolvent condition $\left\|(\lambda-T(z))^{-1}\right\| \leq M /(|\lambda|-1)$ for all $|\lambda|>1$. Similarly, the set $\mathcal{K}_{Q}^{\infty}$ relates to the iterated Kreiss condition $\left\|(\lambda-T(z))^{-k}\right\| \leq M /(|\lambda|-1)^{k}$ for $k \in \mathbb{N}$. For the Ritt set we have $z \in \mathcal{R}_{Q}$ if and only if $\left\|(\lambda-T(z))^{-1}\right\| \leq M /(|\lambda-1|)$ for all $|\lambda|>1$. Out of the Tauberian sets $\mathcal{T}_{Q}^{\alpha}$, only the cases $\alpha=1 / 2$ and $\alpha=1$ will be studied in this paper, and they correspond to the differences of consecutive powers of $T(z)$.

Before going any further, let us give examples:

Remark 2.1. Suppose $Q=\left[\begin{array}{ll}0 & 1 \\ 0 & 0\end{array}\right]$. We get $(1+z Q)^{k}=\left[\begin{array}{cc}1 & k z \\ 0 & 1\end{array}\right],(1-z Q)^{-k}=(1+$ $z Q)^{k}$, and $e^{z Q}=\left[\begin{array}{ll}1 & z \\ 0 & 1\end{array}\right]$. It follows $\mathcal{A}_{Q}^{k}=\mathcal{B}_{Q}=\mathcal{G}_{Q}=\{0\}$ for any $k$. Moreover, $z Q(1+z Q)^{k}=\left[\begin{array}{ll}0 & z \\ 0 & 0\end{array}\right]$, and thus $\mathcal{T}_{Q}=\mathcal{T}_{Q}^{1 / 2}=\{0\}$.

The higher dimensional Jordan matrices and even all algebraic (quasi)nilpotent operators have exactly the same properties:

Proposition 2.2. Let $Q \in \mathcal{L}(X), Q \neq 0$, be a nilpotent operator. Then $\mathcal{A}_{Q}=$ $\mathcal{B}_{Q}=\mathcal{G}_{Q}=\mathcal{T}_{Q}^{1 / 2}=\{0\}$.

Proof. If $Q^{n+1}=0, Q^{n} \neq 0$, and $j>n+1$, we have $(I-T(z)) T(z)^{j}=-z Q(1+$ $z Q)^{j}=-\sum_{k=1}^{n}\left(\begin{array}{c}j \\ k-1\end{array}\right) z^{k} Q^{k}$. Now

$$
\lim _{j \rightarrow \infty} \frac{\left(\begin{array}{c}
j \\
n-1
\end{array}\right)}{\sum_{k=1}^{n}\left(\begin{array}{c}
j \\
k-1
\end{array}\right)}=1, \quad \text { and hence } \quad \lim _{j \rightarrow \infty} \frac{\sum_{k=1}^{n}\left(\begin{array}{c}
j \\
k-1
\end{array}\right) z^{k} Q^{k}}{\sum_{k=1}^{n}\left(\begin{array}{c}
j \\
k-1
\end{array}\right)}=z^{n} Q^{n}
$$


by convex combinations. We conclude from this that

$$
\lim _{j \rightarrow \infty} \frac{(I-T(z)) T(z)^{j} x}{\sum_{k=1}^{n}\left(\begin{array}{c}
j \\
k-1
\end{array}\right)}=z^{n} Q^{n} x \quad \text { for all } \quad x \in X
$$

which gives the estimate

$$
\left\|(I-T(z)) T(z)^{j} x\right\|>\frac{1}{2} \sum_{k=1}^{n}\left(\begin{array}{c}
j \\
k-1
\end{array}\right)\left\|z^{n} Q^{n} x\right\|>\frac{1}{2}\left(\begin{array}{c}
j \\
n-1
\end{array}\right)\left\|z^{n} Q^{n} x\right\|
$$

for all $j$ large enough. If $Q^{n} x \neq 0$ and $z \neq 0$, we conclude from (2.7) that $z \notin \mathcal{T}_{Q}^{1 / 2}$ since $\left(\begin{array}{c}j \\ n-1\end{array}\right)$ is a polynomial of degree $n-1$ in variable $j$. We have now proved $\mathcal{T}_{Q}^{1 / 2}=\{0\}$ which implies $\mathcal{B}_{Q}=\{0\}$ by claim (viii) of Theorem 2.9. To prove the remaining claims, it is sufficient (by the same theorem) to treat $\mathcal{A}_{Q}$ in a similar manner.

Remark 2.3. Let us describe the sets (2.1) - (2.6) in the case $Q=-V^{\alpha}$ where

$$
\left(V^{\alpha} f\right)(x):=\frac{1}{\Gamma(\alpha)} \int_{0}^{x}(x-v)^{\alpha-1} f(v) d v
$$

is the quasinilpotent Riemann-Liouville operator on $L^{2}(0,1)$ for $\alpha \in(0,1]$. When $\alpha=1$ we have the Volterra operator that satisfies

$$
\mathcal{B}_{Q}=\mathcal{G}_{Q}=\mathcal{T}_{Q}^{1 / 2}=\overline{\mathbb{R}}_{+}, \quad \mathcal{A}_{Q}^{k}=\mathbb{C}_{+}, \quad \text { and } \quad \mathcal{T}_{Q}=\mathcal{R}_{Q}=\{0\}
$$

see [Tse03, Theorem 1], [Lyu10, Theorem 1.1] as well as Theorem 2.9 below. Further examples of quasinilpotent operators are given by $\left(V_{\phi} f\right)(x)=(V f)(\phi(x))$ for $\phi \in$ $C[0,1]$, in which case $V_{\phi}$ is quasinilpotent if and only if $\phi(x) \leq x$ for all $x \in[0,1]$; see [Ton89] and [Whi87]. For $\alpha \in(0,1)$, we have $\overline{\mathbb{R}}_{+} \subset \mathcal{R}_{Q}$; see [Lyu01, p. 137] and also [Dun08a]. This provides us with an example of a bounded analytic semigroup generated by a quasinilpotent operator.

We give next the elementary properties of the sets defined in (2.6)-(2.2) based on a direct application of well-known results.

Proposition 2.4. Let $Q \in \mathcal{L}(X)$ be quasinilpotent. Then the following holds:

(i) The sets $\mathcal{B}_{Q}$ and $\mathcal{G}_{Q}$ are convex.

(ii) The sets $\mathcal{B}_{Q}$ and $\mathcal{K}_{Q}^{\infty}$ are star-like.

(iii) $\mathcal{A}_{Q}^{k} \mathbb{R}_{+}=\mathcal{A}_{Q}^{k}$ for $k \in \mathbb{N}$ and $\mathcal{G}_{Q} \mathbb{R}_{+}=\mathcal{G}_{Q}$; i.e., they consist of full rays.

Proof. Since convex combinations of power bounded operators are power-bounded, we have $\alpha z_{1}+\beta z_{2} \in \mathcal{B}_{Q}$ if $z_{1}, z_{2} \in \mathcal{B}_{Q}$ and $\alpha, \beta \geq 0$ satisfy $\alpha+\beta=1$. That $\mathcal{B}_{Q}$ is star-like follows from convexity and the fact that $0 \in \mathcal{B}_{Q}$. The full ray property of the sets is trivial as well as convexity of $\mathcal{G}_{Q}$.

It remains to prove that $\mathcal{K}_{Q}^{\infty}$ is star-like. Using the Hille-Yoshida generator theorem we see that each $e^{i \phi}-1+e^{i \phi} z Q$ for $\phi \in[-\pi, \pi)$ generates a bounded semigroup (with an upper bound $M_{z}$ not depending on $\phi$ ) if and only if $z \in \mathcal{K}_{Q}^{\infty}$; see [Nev97, p. 248-249]. For $\alpha \in[0,1]$ and $t \geq 0$ we have

$$
\left\|e^{t\left(e^{i \phi}-1+e^{i \phi} \alpha z Q\right)}\right\| \leq e^{-t(1-\alpha)(1-\cos \phi)}\left\|e^{t\left(e^{i \phi}-1+e^{i \phi} z Q\right)}\right\| \leq M_{z}
$$

and the claim follows. 
Remark 2.5. We conclude that $\mathcal{G}_{Q}$ is a convex sets consisting of full rays, i.e., a sector, a single ray, or just the set $\{0\}$. Thus, either $\mathcal{G}_{Q} \subset e^{i \phi} \overline{\mathbb{C}}_{+}$for some $\phi \in[-\pi, \pi)$ or $\mathcal{G}_{Q}=\mathbb{C}$ which implies the boundedness of the entire function $e^{t Q}$; hence $Q=0$ by the Liouville's theorem.

The Hille-Yoshida generator theorem for continuous semigroups takes the following form:

Proposition 2.6. Defining the operators $T_{z}$ for $z \in \mathbb{C}$ by (1.3), we have

$$
\mathcal{G}_{Q}=\left\{z \in \mathbb{C}: \sup _{s>0, k \in \mathbb{N}}\left\|T_{s z}^{k}\right\|<\infty\right\}=\left\{z \in \mathbb{C}: \limsup _{s \rightarrow+\infty}\left(\sup _{k \in \mathbb{N}}\left\|T_{s z}^{k}\right\|\right)<\infty\right\} .
$$

This Gelfand-Hille theorem is a consequence of Proposition 2.6:

Proposition 2.7. Let $Q \in \mathcal{L}(X)$ be a quasinilpotent operator. Suppose there is $z \in \mathcal{G}_{Q}, z \neq 0$, such that $-z \in \mathcal{T}_{Q}^{1 / 2}$. Then $Q=0$.

The conclusion can be written as $\left(-\mathcal{G}_{Q}\right) \cap \mathcal{T}_{Q}^{1 / 2}=\{0\}$ for $Q \neq 0$.

Proof. We clearly have

$$
\|I-T(-z)\| \leq\left\|T(-z)^{-j}\right\| \cdot\left\|(I-T(-z)) T(-z)^{j}\right\| .
$$

Now $T(-z)^{-1}=(I-z Q)^{-1}=T_{z}$. If $z \in \mathcal{G}_{Q}$, then $\left\|T_{z}^{j}\right\| \leq M<\infty$ for all $j>0$. If $-z \in \mathcal{T}_{Q}^{1 / 2}$, we have $\left\|(I-T(-z)) T(-z)^{j}\right\| \leq \frac{C}{\sqrt{j+1}}$ for all $j>0$. Then

$$
\|I-T(-z)\| \leq \frac{M C}{\sqrt{j+1}} \rightarrow 0 \quad \text { as } j \rightarrow \infty ;
$$

hence $T(-z)=I$, and $Q=0$ follows if $z \neq 0$.

We remark that the classical Gefand-Hille theorem (see [Zem94, Theorem 1])) implies that neither of the sets $\mathcal{B}_{Q}$ or $\mathcal{G}_{Q}$ contains a line $e^{i \phi} \mathbb{R}$ for $\phi \in[-\pi / 2, \pi / 2)$ unless $Q=0$. Indeed, if $\mathbb{R} \subset \mathcal{G}_{Q}$ then $T:=e^{Q}$ would be an operator with $\sigma(T)=\{1\}$ and $\sup _{k \in \mathbb{Z}}\left\|T^{k}\right\|<\infty$. The claim about $\mathcal{B}_{Q}$ follows from the inclusion $\mathcal{B}_{Q} \subset \mathcal{G}_{Q}$ given in Theorem 2.9 below.

Proposition 2.8. The following are equivalent for $z=r e^{i \theta} \in \mathbb{C}$ :

(i) There exists a constant $C<\infty$ such that

$$
\left\|(1-s z Q)^{-1}\right\| \leq C \quad \text { for all } s \text { with } \operatorname{Re} s>-1 / 2 .
$$

(i.e., $z \in \mathcal{R}_{Q}$ )

(ii) There exists $\delta>0$ with the following property: For any $\eta \in[0, \delta)$, there exists a constant $C_{\eta}<\infty$ such that

$$
\left\|(1-s z Q)^{-1}\right\| \leq C_{\eta} \quad \text { for all } \quad s \in \Sigma_{\pi / 2+\eta} \cup\{\operatorname{Re} s>-1 / 2\} .
$$

(iii) There exists $\delta>0$ with the following property: For any $\eta \in[0, \delta)$, there exists a constant $C_{\eta}<\infty$ such that

$$
\left\|(1-s Q)^{-1}\right\| \leq C_{\eta} \quad \text { for all } \quad s \in e^{i \theta} \Sigma_{\pi / 2+\eta} \cup\left\{\operatorname{Re}\left(e^{-i \theta} s\right)>-r / 2\right\} .
$$

(iv) $T(z)=I+z Q$ is power-bounded, and it satisfies the Tauberian condition $\sup _{n \in \mathbb{N}}(n+1)\left\|(I-T(z)) T(z)^{n}\right\|<\infty$. 
This is plainly the characterisation of Ritt's operators in [MNY09, Proposition 1.1] when applied to $T(z)$ in (1.3).

There is a number of inclusions that the sets in (2.1) - (2.6) satisfy:

Theorem 2.9. Let $Q \in \mathcal{L}(X)$ be quasinilpotent. Then the following holds:

(i) $\mathcal{R}_{Q}=\mathcal{B}_{Q} \cap \mathcal{T}_{Q}$;

(ii) $\mathcal{G}_{Q} \mathbb{C}_{+} \subset \mathcal{A}_{Q}^{k}$ for all $k \in \mathbb{N}$ and, in particular, $\mathcal{R}_{Q} \mathbb{C}_{+} \subset \mathcal{A}_{Q}$;

(iii) $\mathcal{B}_{Q} \subset \mathcal{B}_{Q} \mathbb{R}_{+} \subset \mathcal{G}_{Q} \subset \mathcal{A}_{Q}^{k}$ for all $k \in \mathbb{N}$;

(iv) $\mathcal{R}_{Q}=\mathcal{A}_{Q}^{k} \cap \mathcal{T}_{Q}$ for all $k \in \mathbb{N}$;

(v) $\mathcal{T}_{Q} \subset \mathcal{T}_{Q}^{1 / 2}$ and $\mathcal{G}_{Q} \cap \mathcal{T}_{Q}^{1 / 2} \subset \mathcal{B}_{Q}$;

(vi) $\mathcal{R}_{Q}=\mathcal{G}_{Q} \cap \mathcal{T}_{Q}$

(vii) $\mathcal{R}_{Q} \mathbb{R}_{+}=\mathcal{R}_{Q}$,

(viii) $\mathcal{B}_{Q}[0,1) \subset \mathcal{T}_{Q}^{1 / 2}$

(ix) $\mathcal{B}_{Q} \subset \mathcal{K}_{Q}$ and $\mathcal{K}_{Q} \mathbb{C}_{+} \subset \mathcal{A}_{Q}$,

(x) $\mathcal{B}_{Q} \subset \mathcal{K}_{Q}^{\infty} \subset \mathcal{G}_{Q} \cap \mathcal{K}_{Q}$, and

(xi) $\left(-\mathcal{B}_{Q^{2}}\right)^{1 / 2} \cap \mathcal{G}_{Q} \subset \mathcal{B}_{Q}$.

Proof. Claim (i) and the latter part of (ii) follow directly from Proposition 2.8. The first inclusion of (ii) follows from the Hille-Yosida theorem: if $z \in \mathcal{G}_{Q}$, then for all $s \in \mathbb{C}_{+}$and $k \in \mathbb{N}$ we have $\left\|\left(s^{-1}-z Q\right)^{-k}\right\| \leq M /\left(\operatorname{Re} s^{-1}\right)^{k}$; i.e., $\left\|(I-s z Q)^{-k}\right\| \leq$ $M / \cos ^{k} \phi$ for some constant $M<\infty$ where $\cos \phi=\operatorname{Re} s /|s|$. Claim (iii) follows from the fact that the power series coefficients of $e^{x}$ are positive, together with (2.9) and the fact that $\mathcal{G}_{Q} \mathbb{R}_{+}=\mathcal{G}_{Q}$ by Proposition 2.4. Let us prove the first part of claim (iv). The inclusion $\mathcal{A}_{Q} \cap \mathcal{T}_{Q} \subset \mathcal{B}_{Q}$ follows by applying [MNY09, Theorem 1] to operators $1+z Q$. Hence $\mathcal{A}_{Q} \cap \mathcal{T}_{Q} \subset \mathcal{B}_{Q} \cap \mathcal{T}_{Q}=\mathcal{R}_{Q}$ by claim (i). The converse inclusion follows from claims (i) and (iii).

Claim (v) is another tauberian theorem, and it follows from [Pey69, Theorem III.5 on p. 68] as pointed out in [MNY09].

Claim (vii) is given in [Tse03, Proposition 2] but we prove it here, too. Let $z=r e^{i \theta} \in \mathcal{R}_{Q}$ and $h>0$ be given. Then there exists $\delta>0$ such that $Q$ satisfies condition (iii) of Proposition 2.8. This is equivalent with having $\left\|(1-s \cdot h Q)^{-1}\right\| \leq$ $C_{\theta, \delta}$ for any $\eta \in[0, \delta)$ and all $s \in e^{i \theta} \Sigma_{\pi / 2+\eta} \cup\left\{\operatorname{Re}\left(e^{-i \theta} s\right)>-r / 2 h\right\}$ since $h^{-1} e^{i \theta} \Sigma_{\pi / 2+\eta}=e^{i \theta} \Sigma_{\pi / 2+\eta}$ and $h^{-1}\left\{\operatorname{Re}\left(e^{-i \theta} s\right)>-r / 2\right\}=\left\{\operatorname{Re}\left(e^{-i \theta} s\right)>-r / 2 h\right\}$. If $h>1$, we do not have the inclusion $\left\{\operatorname{Re}\left(e^{-i \theta} s\right)>-r / 2\right\} \subset\left\{\operatorname{Re}\left(e^{-i \theta} s\right)>-r / 2 h\right\}$ but it is nevertheless easy to see that the estimate $\left\|(1-s \cdot h Q)^{-1}\right\| \leq C_{\theta, \delta}^{\prime}$ holds (with a larger constant $C_{\theta, \delta}^{\prime}<\infty$ in place of $C_{\theta, \delta}$ ) even for $s$ in the larger set $e^{i \theta} \Sigma_{\pi / 2+\eta} \cup\left\{\operatorname{Re}\left(e^{-i \theta} s\right)>-r / 2\right\}$ since this set differs from $e^{i \theta} \Sigma_{\pi / 2+\eta} \cup\left\{\operatorname{Re}\left(e^{-i \theta} s\right)>\right.$ $-r / 2 h\}$ only by a precompact set. Proposition 2.8 proves now claim (i).

Claim (viii) follows from the fact that for $\alpha \in[0,1)$ and power-bounded $T$, the bound $\sqrt{j+1}\left\|\left(I-T_{\alpha}\right) T_{\alpha}^{j}\right\| \leq M_{\alpha}<\infty$ holds for all operators $T_{\alpha}=\alpha+(1-\alpha) T$, see [Nev93, Theorem 4.5.3]. See also [Tse03, Remark 2] and [FW73, Lemma 2.1].

Let us prove claim (ix). The inclusion $\mathcal{B}_{Q} \subset \mathcal{K}_{Q}$ follows because power-boundedness implies (even the iterated) the Kreiss resolvent condition by a straightforward argument. Let us prove the latter inclusion. Clearly for all $s \in \mathbb{C}$ we have $|s+1|-|s|=\frac{2 \operatorname{Re} s+1}{|s+1|+|s|}$, and $|s|<|s+1|$ for $s \in \mathbb{C}_{+}$. Thus for $s \in \mathbb{C}_{+}$we have $\cos \theta-\frac{1}{2|s+1|}<|s+1|-|s|<\cos \phi+\frac{1}{2|s|}$ where $\cos \theta=\frac{\operatorname{Re}(s+1)}{|s+1|}>\frac{\operatorname{Re} s}{|s|}=\cos \phi$ 
and $0 \leq \phi<\theta$. It follows that for $\phi \in[0, \pi / 2)$, there are costants $m_{\phi}, M_{\phi}$ such that

$$
0<m_{\phi} \leq|s+1|-|s|<M_{\phi}<\infty
$$

for all $s \in \Sigma_{\phi}$.

Now, let $z \in \mathcal{K}_{Q}$ and $\phi \in[0, \pi / 2)$. Then for any $\alpha \in[-\phi, \phi]$ we get

$$
\sup _{r \geq 0}\left\|\left(1-r \cdot e^{i \alpha} z Q\right)^{-1}\right\| \leq \sup _{s \in \Sigma_{\phi}}\left\|(1-s z Q)^{-1}\right\|<\infty
$$

by equation (2.12). Thus $e^{i \alpha} z \in \mathcal{A}_{Q}$ and also $\Sigma_{\phi} z \subset \mathcal{A}_{Q}$. Because $z \in \mathcal{K}_{Q}$ and $\phi \in(-\pi / 2, \pi / 2)$ are arbitrary, we conclude that $\mathcal{K}_{Q} \mathbb{C}_{+} \subset \mathcal{A}_{Q}$.

The nontrivial part $\mathcal{K}_{Q}^{\infty} \subset \mathcal{G}_{Q}$ of claim (x) follows from the estimate $\left\|e^{t e^{i \phi} z Q}\right\| \leq$ $M_{z} e^{t(1-\cos \phi)}$ for all $\phi \in[-\pi, \pi)$ that can be proved in a similar way as (2.8).

Finally claim (xi): Note that (1.3) implies $T(z)^{k}=\left(1-z^{2} Q^{2}\right)^{k} T_{z}^{k}$ for all $k \in \mathbb{N}$. If $z^{2} \in-\mathcal{B}_{Q^{2}}$, then $1-z^{2} Q^{2}$ is power-bounded. Since $z \in \mathcal{G}_{Q}$, the operator $T_{z}$ is power-bounded, and thus $z \in \mathcal{B}_{Q}$.

Remark 2.10. Claim (i) is strengthened in Theorem 3.3. It is instructive to compare claims (ii) and (ix). By claim (viii) we get $\mathcal{B}_{Q}^{\circ} \subset \mathcal{T}_{Q}^{1 / 2}$ where $\mathcal{B}_{Q}^{\circ}$ denotes the open interior of $\mathcal{B}_{Q}$. A stronger form of this claim is given by Proposition 3.2.

Remark 2.11. Note that (ii) and (iv) imply that the inclusion $\mathcal{R}_{Q}=\mathcal{A}_{Q} \cap \mathcal{T}_{Q} \subset \mathcal{A}_{Q}$ is strict if $\mathcal{R}_{Q}$ has a nonempty interior. Hence the inclusion $\mathcal{A}_{Q} \subset \mathcal{T}_{Q}$ does not generally hold, and even $\mathcal{A}_{Q} \subset \mathcal{T}_{Q}^{1 / 2}$ fails by the remark following Theorem 6.3. It is an open question whether the converse inclusion $\mathcal{T}_{Q}^{1 / 2} \subset \mathcal{A}_{Q}$ always holds. This and the stronger inclusion $\mathcal{T}_{Q} \subset \mathcal{B}_{Q}$ are given in Theorem 6.3 under quite restrictive assumptions.

Corollary 2.12. For a quasinilpotent $Q \in \mathcal{L}(X)$ we have $\mathcal{B}_{Q}=\mathcal{G}_{Q}$ if and only if $\mathcal{G}_{Q} \subset \mathcal{T}_{Q}^{1 / 2}$.

Indeed, this follows from claims (iii) and (v) of Theorem 2.9.

A slight improvement of Proposition 2.8 is possible:

Proposition 2.13. For any quasinilpotent $Q \in \mathcal{L}(X)$ we have

$$
\mathcal{R}_{Q}=\left\{z \in \mathbb{C}: \sup _{\operatorname{Re} s>0}\left\|(1-s z Q)^{-1}\right\|<\infty\right\} .
$$

Proof. It is clear by continuity that $\sup _{\operatorname{Re} s>0}\left\|(1-s z Q)^{-1}\right\|=\sup _{\operatorname{Re} s \geq 0}\left\|(1-s z Q)^{-1}\right\|$. By (2.5), it is thus enough to show that

$$
A:=\left\{z \in \mathbb{C}: \sup _{\operatorname{Re} s \geq 0}\left\|(1-s z Q)^{-1}\right\|<\infty\right\} \subset \mathcal{R}_{Q} .
$$

Fix $z \in A$, and define $M:=\sup _{\operatorname{Re} s \geq 0}\left\|(I-s z Q)^{-1}\right\|<\infty$ and $\alpha:=\frac{1}{2 M\|z Q\|}>0$. Take any $s=x+y i \in \mathbb{C}$ where $x \in(-\alpha, 0]$ and $y \in \mathbb{R}$. Now

$$
\left\|(I-s z Q)^{-1}\right\| \leq\left\|(I-i y z Q)^{-1}\right\| \cdot\left\|\left(I-(I-i y z Q)^{-1}(s-i y) z Q\right)^{-1}\right\|
$$

where $\left\|(I-i y z Q)^{-1}\right\|<M$ and

$$
\left\|(I-i y z Q)^{-1}(s-i y) z Q\right\| \leq M \alpha\|z Q\|=\frac{1}{2} .
$$

It follows from this that

$$
\left\|(I-s z Q)^{-1}\right\| \leq M_{\alpha} \quad \text { for all } s \text { satisfying }-\alpha<\operatorname{Re} s \leq 0
$$


holds with $M_{\alpha}=2 M$, and hence for all $s$ with Re $s>-\alpha$ because $z \in A$. Condition (2.14) for $\operatorname{Re} s>-\alpha$ is clearly equivalent with

$$
\left\|\left(I-\frac{s}{2 \alpha} \cdot 2 \alpha z Q\right)^{-1}\right\|<M_{\alpha} \quad \text { for all } s \text { satisfying } \operatorname{Re} \frac{s}{2 \alpha}>-1 / 2 .
$$

which by (2.5) is equivalent with $2 \alpha z \in \mathcal{R}_{Q}$, and further equivalent with $z \in \mathcal{R}_{Q}$ by claim (vii) of Theorem 2.9 .

Clearly (2.13) implies trivially $\mathcal{R}_{Q} \mathbb{R}_{+} \subset \mathcal{R}_{Q}$ but we use it in the above proof. Using the sectorial extension property in claim (ii) of Proposition 2.8 and the boundedness of the resolvent in any compact set, we see that whenever $z \in \mathcal{R}_{Q}$ holds, we have

$$
\sup _{\operatorname{Re} s>\alpha}\left\|(I-s z Q)^{-1}\right\|<\infty \quad \text { for all } \quad \alpha<0 .
$$

The resolvent estimation technique in the proof of Proposition 2.13 shows also the following: If $M_{\alpha}:=\sup _{\operatorname{Re} s>\alpha}\left\|(I-s z Q)^{-1}\right\|<\infty$ for some $\alpha>0$, then $M_{\beta}<\infty$ holds for some $\beta \in(0, \alpha)$. Indeed, for any $\alpha>0$ it follows from $\sup _{\operatorname{Re} s>\alpha}\left\|(I-s z Q)^{-1}\right\|<\infty$ by continuity that $\sup _{\operatorname{Re} s \geq \alpha}\left\|(I-s z Q)^{-1}\right\|<\infty$. This implies $\sup _{\operatorname{Re} s>\beta}\left\|(I-s z Q)^{-1}\right\|<\infty$ for some $\beta \in(0, \alpha)$ by an estimation using the resolvent identity. However, we cannot exclude the possibilities that $\gamma:=\inf \left\{\beta: M_{\beta}<\infty\right\}>0$ or $M_{\gamma}=\infty$ even in the case when $\gamma=0$.

An interpolation between $\mathcal{R}_{Q}$ and $\mathcal{T}_{Q}^{1 / 2}$ produces the following result:

Proposition 2.14. For a quasinilpotent $Q \in \mathcal{L}(X)$ we have

$$
\left\{\alpha z_{1}+(1-\alpha) z_{2}: \alpha \in[0,1], z_{1} \in \mathcal{T}_{Q}^{1 / 2}, z_{2} \in \mathcal{R}_{Q}\right\} \subset \mathcal{T}_{Q}^{1 / 2} .
$$

Since always $0 \in \mathcal{R}_{Q}$, we conclude that $\mathcal{T}_{Q}^{1 / 2}$ is a star-like set.

Proof. Fix $z_{1} \in T_{Q}^{1 / 2}, z_{2} \in \mathcal{R}_{Q}$, and $\alpha \in[0,1]$. Then there are constants $0<$ $M_{1}, M_{2}, M_{3}, M_{4}<\infty$ such that the estimates

$$
\begin{aligned}
& \left\|z_{1} Q T\left(z_{1}\right)^{j}\right\| \leq \frac{M_{1}}{\sqrt{j+1}}, \quad\left\|T\left(z_{1}\right)^{j}\right\| \leq M_{2} \sqrt{j+1} \\
& \left\|z_{2} Q T\left(z_{2}\right)^{k-j}\right\| \leq \frac{M_{3}}{k-j+1}, \text { and }\left\|T\left(z_{2}\right)^{k-j}\right\| \leq M_{4}
\end{aligned}
$$

hold for all $j, k$ satisfying $0 \leq j \leq k$. For the second estimate, it is enough to estimate the sum $I-T\left(z_{1}\right)^{j}=\sum_{l=0}^{j-1}\left(I-T\left(z_{1}\right)\right) T\left(z_{1}\right)^{l}$ using the first inequality in (2.15) to obtain $\left\|T\left(z_{1}\right)^{j}\right\| \leq 1+2 M_{1}(\sqrt{j+1}-1)$. The latter two estimates follow from claim (i) of Theorem 2.9 .

Now, define $\tilde{T}(\alpha):=T\left(\alpha z_{1}+(1-\alpha) z_{2}\right)=\alpha T\left(z_{1}\right)+(1-\alpha) T\left(z_{2}\right)$ for $\alpha \in[0,1]$. For all $k$ we have

$$
\begin{aligned}
-(I-\tilde{T}(\alpha)) \tilde{T}(\alpha)^{k} & =\sum_{j=0}^{k}\left(\begin{array}{c}
k \\
j
\end{array}\right) \alpha^{j+1}\left[z_{1} Q T\left(z_{1}\right)^{j}\right](1-\alpha)^{k-j} T\left(z_{2}\right)^{k-j} \\
& +\sum_{j=0}^{k}\left(\begin{array}{c}
k \\
j
\end{array}\right) \alpha^{k} T\left(z_{1}\right)^{j}(1-\alpha)^{k-j+1}\left[z_{2} Q T\left(z_{2}\right)^{k-j}\right] .
\end{aligned}
$$


By using the estimates (2.15) we get

$$
\begin{aligned}
\left\|(I-\tilde{T}(\alpha)) \tilde{T}(\alpha)^{k}\right\| & \leq M_{1} M_{4} \sum_{j=0}^{k} \frac{1}{\sqrt{j+1}}\left(\begin{array}{c}
k \\
j
\end{array}\right) \alpha^{j+1}(1-\alpha)^{k-j} \\
& +M_{2} M_{3} \sum_{j=0}^{k} \frac{\sqrt{j+1}}{k-j+1}\left(\begin{array}{c}
k \\
j
\end{array}\right) \alpha^{j}(1-\alpha)^{k-j+1} .
\end{aligned}
$$

Note that $\sqrt{\frac{k+1}{j+1}}\left(\begin{array}{l}k \\ j\end{array}\right)=\sqrt{\frac{j+1}{k+1}}\left(\begin{array}{c}k+1 \\ j+1\end{array}\right) \leq\left(\begin{array}{c}k+1 \\ j+1\end{array}\right)$ and $\frac{\sqrt{(k+1)(j+1)}}{k-j+1}\left(\begin{array}{l}k \\ j\end{array}\right)=$ $\sqrt{\frac{j+1}{k+1}}\left(\begin{array}{c}k+1 \\ j\end{array}\right) \leq\left(\begin{array}{c}k+1 \\ j\end{array}\right)$ for all $0 \leq j \leq k$. By the binomial theorem, we get $\sqrt{k+1}\left\|(I-\tilde{T}(\alpha)) \tilde{T}(\alpha)^{k}\right\| \leq M_{1} M_{4}\left(1-(1-\alpha)^{k+1}\right)+M_{2} M_{3}\left(1-\alpha^{k+1}\right)$.

Remark 2.15. Using the same technique as in the proof of Proposition 2.14, we get the estimate $\left\|(I-T(z)) T(z)^{j}\right\| \leq M \ln (j+1) / \sqrt{j+1}$ where $z=\alpha z_{1}+(1-\alpha) z_{2}$ for $\alpha \in[0,1], z_{1} \in \mathcal{T}_{Q}$, and $z_{2} \in \mathcal{B}_{Q}$. Similarly, we have $\left\|(I-T(z)) T(z)^{j}\right\| \leq$ $M \ln (j+1) /(j+1)$ if $z_{1} \in \mathcal{T}_{Q}$, and $z_{2} \in \mathcal{R}_{Q}$ instead. Hence, if $z_{1} \in \mathcal{T}_{Q} \backslash \mathcal{A}_{Q}$ and $z_{2} \in \mathcal{R}_{Q} \subset \mathcal{A}_{Q}$ we have at most logarithmic growth in the powers $T(z)^{j}$ for those $z=\alpha z_{1}+(1-\alpha) z_{2}, \alpha \in(0,1]$, that satisfy $z \in \mathcal{A}_{Q}$; this follow by modifying the proof of Tauberian theorem [MNY09, Theorem 1].

Note that in the proof of Proposition 2.14, we use for $z \in \mathcal{T}_{Q}^{1 / 2}$ the estimate $\left\|T(z)^{j}\right\| \leq M \sqrt{j+1}$ which cannot be improved by the Tauberian approach used in [MNY09, Theorem 1] even if $z \in \mathcal{A}_{Q} \cap \mathcal{T}_{Q}^{1 / 2}$.

\section{INTERIOR POINTS OF $\mathcal{B}_{Q}$ AND $\mathcal{R}_{Q}$}

Recall that $\mathcal{B}_{Q}^{\circ}$ denotes the open interior of $\mathcal{B}_{Q}$.

Proposition 3.1. The function $\mathcal{B}_{Q}^{\circ} \ni z \mapsto N_{z} \in \mathbb{R}_{+}$defined by

$$
N_{z}:=\sup _{n \geq 1}\left\|(1+z Q)^{n}\right\|
$$

is uniformly bounded on compact subsets of $\mathcal{B}_{Q}^{\circ}$.

Proof. Let $z_{0} \in \mathcal{B}_{Q}^{\circ}$ be arbitrary, and define $\delta=\frac{1}{2} \operatorname{dist}\left(z_{0}, \partial \mathcal{B}_{Q}\right)$. Define $D=\{z \in$ $\left.\mathbb{C}:\left|z-z_{0}\right|<\delta\right\}$

Then there exists a regular, convex polygon around $D$ of, say, $n$ vertices $v_{1}, v_{2}, \ldots, v_{n}$ such that

$$
D \subset \operatorname{conv}\left\{v_{1}, v_{2}, \ldots, v_{n}\right\} \subset \mathcal{B}_{Q}^{\circ}
$$

where conv denotes the closed, convex hull. Now, the set $\operatorname{conv}\left\{v_{1}, v_{2}, \ldots, v_{m}\right\}$ can be written as a union of $m-2$ closed triangles whos vertices are in the set $\left\{v_{1}, v_{2}, \ldots, v_{m}\right\}$. To show that $z \mapsto N_{z}$ is uniformly bounded on $D$, it is enough to show that the same function is bounded on all closed triangles inside $\mathcal{B}_{Q}^{\circ}$.

So, let $v_{1}, v_{2}, v_{3}$ be the verteces of a triangle, and define $M=\max \left(M_{v_{1}}, M_{v_{2}}, M_{v_{3}}\right)$ (see $(3.1))$. Recall the trinomial formula $(a+b+c)^{n}=\sum_{j, k=0}^{n} c_{j, k}^{n} a^{j} b^{k} c^{n-j-k}$ where the coefficients $c_{j, k}^{n}$ are natural numbers. We have for any $\alpha, \beta, \gamma \in[0,1]$, 
$\alpha+\beta+\gamma=1$, and $n \in \mathbb{N}$ the estimate

$$
\begin{aligned}
& \left\|\left(1+\left(\alpha v_{1}+\beta v_{2}+\gamma v_{3}\right) Q\right)^{n}\right\|=\left\|\left(\alpha\left(1+v_{1} Q\right)+\beta\left(1+v_{2} Q\right)+\gamma\left(1+v_{3} Q\right)\right)^{n}\right\| \\
& \leq \sum_{j, k=0}^{n} c_{j, k}^{n} \alpha^{j} \beta^{k} \gamma^{n-j-k}\left\|\left(1+v_{1} Q\right)^{j}\right\| \cdot\left\|\left(1+v_{2} Q\right)^{k}\right\| \cdot\left\|\left(1+v_{3} Q\right)^{n-j-k}\right\| \\
& \leq M^{3}(\alpha+\beta+\gamma)^{n}=M^{3} .
\end{aligned}
$$

Thus $\sup _{z \in \operatorname{conv}\left(v_{1}, v_{2}, v_{3}\right)} N_{z} \leq M^{3}<\infty$. We have now proved that $z \mapsto N_{z}$ is uniformly bounded on any disk $D$ whose closure is in $\mathcal{B}_{Q}^{\circ}$. The proof is completed by a usual covering argument.

Proposition 3.2. Always $\mathcal{B}_{Q}^{\circ} \subset \mathcal{T}_{Q}$ for any quasinilpotent $Q \in \mathcal{L}(X)$.

Proof. By the Cauchy integral, we have

$$
f^{\prime}\left(z_{0}\right)=\frac{1}{2 \pi i} \int_{\Gamma} f^{\prime}(\xi)\left(\xi-z_{0}\right)^{-1} d \xi=\frac{1}{2 \pi i} \int_{\Gamma} f(\xi)\left(\xi-z_{0}\right)^{-2} d \xi
$$

where $\Gamma$ surrounds $z_{0}$ inside the domain of analyticity of $f$. If $\Gamma=\Gamma_{\delta}:=\{z \in \mathbb{C}$ : $\left.\left|z-z_{0}\right|=\delta\right\}$, we get the estimate

$$
\left\|f^{\prime}\left(z_{0}\right)\right\| \leq \frac{1}{2 \pi} \sup _{\xi \in \Gamma_{\delta}}\|f(\xi)\| \cdot \frac{1}{\delta^{2}} \cdot 2 \pi \delta=\frac{1}{\delta} \sup _{\xi \in \Gamma_{\delta}}\|f(\xi)\|
$$

Note that $(n+1) z Q(1+z Q)^{n}=z \frac{d}{d z}(1+z Q)^{n+1}$ for all $z \in \mathbb{C}$. Applying the above estimate gives for all $z \in \mathcal{B}_{Q}^{\circ}$ and $n \in \mathbb{N}$

$$
(n+1)\left\|z Q(1+z Q)^{n}\right\| \leq|z| \cdot \sup _{\xi \in \Gamma_{\delta}}\left\|(1+\xi Q)^{n+1}\right\| \leq|z| \cdot \sup _{\xi \in \Gamma_{\delta}} M_{\xi}
$$

where $\delta=\operatorname{dist}\left(z, \partial \mathcal{B}_{Q}^{\circ}\right)$. Because $\Gamma_{\delta}$ is a compact subset of $\mathcal{B}_{Q}^{\circ}$, the claim follows from Proposition 3.1.

An alternative proof for Proposition 3.2 can be based on Proposition 4.4.

Theorem 3.3. Let $Q \in \mathcal{L}(X)$ be a quasinilpotent operator. Then (i) $\mathcal{R}_{Q}=\mathcal{B}_{Q}^{\circ} \cup\{0\}$ and $\partial \mathcal{R}_{Q}=\partial \mathcal{B}_{Q}^{\circ} \cup\{0\}$; (ii) $\mathcal{R}_{Q} \neq\{0\}$ if and only if the set $\mathcal{B}_{Q}^{\circ}$ is a non-empty open sector; and (iii) either $\mathcal{R}_{Q}=\{0\}, \mathcal{R}_{Q}=\mathbb{C}$ (i.e., $Q=0$ ), or there exists $\phi \in[-\pi, \pi$ ) and $\theta \in(0, \pi / 2]$ such that $\mathcal{R}_{Q}=e^{i \phi} \Sigma_{\theta} \cup\{0\}$.

Proof. We know by claim (i) of Theorem 2.9 and Proposition 3.2 that

$$
\mathcal{B}_{Q}^{\circ} \cup\{0\} \subset \mathcal{B}_{Q} \cap \mathcal{T}_{Q}=\mathcal{R}_{Q} \subset \mathcal{B}_{Q}
$$

It follows that $\mathcal{B}_{Q}^{\circ}=\mathcal{R}_{Q}^{\circ}$ where $\mathcal{R}_{Q}^{\circ}$ denotes the open interior of $\mathcal{R}_{Q}$. Because $\mathcal{B}_{Q}$ is convex by Proposition 2.4, so is its interior $\mathcal{B}_{Q}^{\circ}$. Because $\mathcal{R}_{Q}$ consists of full rays by claim (vii) of Theorem 2.9, so does its interior $\mathcal{R}_{Q}^{\circ}$. We conclude that either $\mathcal{R}_{Q}^{\circ}=\emptyset$, or it is an open, convex set that consists of full rays. In other words, $\mathcal{R}_{Q}^{\circ}$ is an open sector if $\mathcal{R}_{Q}^{\circ} \neq \emptyset$. It follows from this that

$$
\mathcal{R}_{Q}=\mathcal{R}_{Q}^{\circ} \cup\{0\} \cup E \quad \text { with } \quad E=\cup_{\phi \in A} e^{i \phi} \mathbb{R}_{+}
$$

where $A \subset[-\pi, \pi), \mathcal{R}_{Q}^{\circ} \cap E=\emptyset$, and $E^{\circ}=\emptyset$. Thus $e^{i \phi} \mathbb{R}_{+} \subset \partial \mathcal{R}_{Q}$ for all $\phi \in A$.

We proceed to show that $\mathcal{R}_{Q}=\mathcal{R}_{Q}^{\circ} \cup\{0\}$ by excluding the set $E$. It is clear that this equality may fail only if $e^{i \phi} \mathbb{R}_{+} \subset \partial \mathcal{R}_{Q} \cap \mathcal{R}_{Q}$ for some $\phi \in[-\pi, \pi)$. This is, however, impossible by the equivalence of (i) and (ii) of Proposition 2.8, implying 
that any ray in $\mathcal{R}_{Q}$ is in fact contained in an open sector in $\mathcal{R}_{Q}$. This proves that $\mathcal{R}_{Q}=\mathcal{B}_{Q}^{\circ} \cup\{0\}$ and hence $\partial \mathcal{R}_{Q}=\partial \mathcal{B}_{Q}^{\circ}$ if $\mathcal{R}_{Q} \neq\{0\}$. This proves (i), and the latter two claims are consequences of this.

Thus, the set $\mathcal{R}_{Q}$ is convex sector whose central angle plays such an important role that it deserves a name:

Definition 3.4. Let $Q \in \mathcal{L}(X)$ be a quasinilpotent operator. If $\mathcal{R}_{Q} \neq\{0\}$, we call the angle $\theta \in(0, \pi / 2]$ the Ritt angle of operator $Q$ if $\mathcal{R}_{Q}=e^{i \phi} \Sigma_{\theta} \cup\{0\}$ for some $\phi \in[-\pi, \pi)$. If $\mathcal{G}_{Q}=e^{i \phi} \mathbb{R}_{+}$for some $\phi \in[-\pi, \pi)$, then we say that the Ritt angle of $Q$ equals 0. (If $\mathcal{G}_{Q}=\{0\}$, the Ritt angle of $Q$ is not defined. )

It is now possible to improve claim (iii) of Theorem 2.9 a bit:

Proposition 3.5. We have $\overline{\mathcal{B}_{Q}} \subset \mathcal{A}_{Q}$ and hence $\partial \mathcal{B}_{Q} \cap \mathcal{T}_{Q}=\{0\}$ for any quasinilpotent operator $Q \in \mathcal{L}(X)$.

Proof. We show first that $\overline{\mathcal{B}_{Q}} \subset \mathcal{A}_{Q}$. This claim clearly holds when $\mathcal{B}_{Q}^{\circ}=\mathbb{C}$ but this situation happens only if $Q=0$ by Remark 2.5 .

Let us first consider the case $\mathcal{B}_{Q}^{\circ}=\emptyset$. Then by convexity, $\mathcal{B}_{Q} \subset e^{i \phi} \overline{\mathbb{R}}_{+}$for some $\phi \in[-\pi, \pi)$. If $\mathcal{B}_{Q}=\{0\}$, there is nothing to prove. Otherwise $\mathcal{B}_{Q}$ is a possibly non-closed, possibly finite interval whose one end is at the origin, and then $\overline{\mathcal{B}_{Q}} \subset \mathcal{B}_{Q} \mathbb{R}_{+} \subset \mathcal{A}_{Q}^{k}$ for all $k \in \mathbb{N}$ by claim (iii) of Theorem 2.9.

Suppose now that $\mathcal{B}_{Q}^{\circ} \neq \emptyset$. By Theorem 3.3, we have $\partial \mathcal{B}_{Q}=\partial \mathcal{R}_{Q}$ (that is a set consisting of two rays), and thus claim (ii) of Theorem 2.9 implies that $\partial \mathcal{B}_{Q} \subset \mathcal{A}_{Q}$ by proximity. We conclude that $\overline{\mathcal{B}_{Q}}=\mathcal{B}_{Q} \cup \partial \mathcal{B}_{Q} \subset \mathcal{A}_{Q}$ as claimed.

We have now shown that $\partial \mathcal{B}_{Q} \subset \mathcal{A}_{Q}$ for all quasinilpotent operators $Q$. If $z$ is in $\partial \mathcal{B}_{Q} \cap \mathcal{T}_{Q}$, then $z \in \mathcal{A}_{Q} \cap \mathcal{T}_{Q}=\mathcal{R}_{Q}$ by claim (iv) of Theorem 2.9. We also have $z \in \partial \mathcal{R}_{Q}$ because $\partial \mathcal{R}_{Q}=\partial \mathcal{B}_{Q}$, and hence $z=0$ follows since $\mathcal{R}_{Q}=\mathcal{B}_{Q}^{\circ} \cup\{0\}$ by Theorem 3.3.

\section{Interior POINTS OF $\mathcal{G}_{Q}, \mathcal{K}_{Q}$, AND $\mathcal{K}_{Q}^{\infty}$}

Let us recall [ABHN01, Definition 3.7.3] of bounded analytic semigroups:

Definition 4.1. We say that a strongly continuous semigroup $S(t), t \in \overline{\mathbb{R}}_{+}$, is a bounded analytic (holomorphic) semigroup of angle $\theta \in(0, \pi / 2]$ if $S(t)$ has a bounded, analytic extension to $\Sigma_{\theta^{\prime}}$ for all $\theta^{\prime} \in(0, \theta)$.

In the context of this paper, the semigroup is given by $S_{z}(t)=e^{t z Q}$ for quasinilpotent $Q$ and $z \in \mathcal{G}_{Q}$. Let us recall a classical result from function theory that we need in proving Theorem 4.3:

Proposition 4.2. Let $X$ be a Banach space. Suppose that $A, \omega, \tau>0, \theta \in(0, \pi)$, and that $\Sigma_{\theta}$ is defined by (1.5). Suppose that th $\mathcal{L}(X)$-valued function $f$ is analytic in $\Sigma_{\theta}$ and continuous in $\overline{\Sigma_{\theta}}$. Suppose that the inequalities

$$
2 \theta<\frac{\pi}{\omega} \quad \text { and } \quad\|f(z)\| \leq A e^{\tau|z|^{\omega}} \text { for all } z \in \Sigma_{\theta}
$$

hold. If $\|f(z)\| \leq 1$ for all $z \in \partial \Sigma_{\theta}$, then also $\|f(z)\| \leq 1$ for all $z \in \Sigma_{\theta}$.

Proof. By considering the functions $z \mapsto\left\langle x^{*}, f(z) x\right\rangle_{X^{*}, X}$ for $x \in X$ and $x^{*} \in X^{*}$, the claim can be reduced to the scalar case which we prove next. Let $\theta<\frac{\pi}{2 \omega}$ and take $z \in \Sigma_{\theta}$. Then for any $\beta$ satisfying $\omega<\beta<\frac{\pi}{2 \theta}$ we have the inequality

$$
\operatorname{Re}(z+1)^{\beta}>|z+1|^{\beta} \delta
$$


where $\delta:=\cos \beta \theta>0$ since $|\arg (z+1)|<|\arg z| \leq \theta$ and $0<\beta \theta<\pi / 2$. Define now $h_{\epsilon}(z)=e^{-\epsilon(z+1)^{\beta}}$ for any $\epsilon>0$. This is an analytic function for $z \in \Sigma_{\theta} \neq \mathbb{C}$, and it is continuous in $\overline{\Sigma_{\theta}}$. Then for all $z \in \overline{\Sigma_{\theta}}$ we have the estimate

$$
\left|f(z) h_{\epsilon}(z)\right| \leq A e^{\tau|z|^{\omega}-\epsilon \operatorname{Re}(z+1)^{\beta}} \leq A e^{\tau|z|^{\omega}-\epsilon \delta|z+1|^{\beta}} \rightarrow 0 \text { as }|z| \rightarrow \infty .
$$

The maximum modulus theorem says that

$$
\max _{z \in \overline{\Sigma_{\theta}}}\left|f(z) h_{\epsilon}(z)\right|=\max _{z \in \partial \Sigma_{\theta}}\left|f(z) h_{\epsilon}(z)\right| \leq \max _{z \in \partial \Sigma_{\theta}}\left|h_{\epsilon}(z)\right|
$$

for all $\epsilon>0$. Letting $\epsilon \rightarrow 0+$ completes the proof.

Note that $f(z)=e^{z}$ satisfies $|f(z)| \leq A e^{\tau|z|^{\omega}}$ for $A=\omega=\tau=1$ and all $z \in C$. If $2 \theta=\pi / \omega=\pi$ and $\Sigma_{\theta}=\mathbb{C}_{+}$, we see that $f$ is bounded on $\partial \Sigma_{\theta}=i \mathbb{R}$ but not on $\Sigma_{\theta}$. Hence the inequality $2 \theta<\frac{\pi}{\omega}$ cannot be replaced by the weaker $2 \theta \leq \frac{\pi}{\omega}$ in Proposition 4.2.

Theorem 4.3. Let $Q \in \mathcal{L}(X)$ be a quasinilpotent operator. Then the following are equivalent for $z \in \mathbb{C}$ :

(i) $z \in \mathcal{G}_{Q}^{\circ}$;

(ii) $z \neq 0$ and the operator $z Q$ generates an analytic semigroup $t \mapsto e^{t z Q}$; and

(iii) $z \in \mathcal{R}_{Q} \backslash\{0\}$.

Thus $\mathcal{R}_{Q}=\mathcal{G}_{Q}^{\circ} \cup\{0\}$. Moreover, we have $\mathcal{R}_{Q}=\{0\}$ if and only if $\mathcal{G}_{Q} \subset e^{i \phi} \overline{\mathbb{R}}_{+}$for some $\phi \in[-\pi, \pi)$.

Proof. (i) $\Rightarrow$ (ii): Take $z \in G_{Q}^{\circ}$, and let $0<\theta<\pi / 2$ such that both $z e^{i \theta}$, $z e^{-i \theta} \in$ $G_{Q}^{\circ}$. It follows that the analytic function $g(t):=e^{t z Q}$ is bounded on both rays $t \in e^{ \pm i \theta} \mathbb{R}_{+}$. Since $\left\|e^{t z Q}\right\| \leq e^{\tau|t|^{\omega}}$ with $\tau=\|z Q\|$ and $\omega=1$, Proposition 4.2 implies now that $\sup _{t \in \Sigma_{\theta}}\|\bar{g}(t)\|<\infty$; hence $z Q$ generates a bounded analytic semigroup with angle $\geq \theta$.

(ii) $\Leftrightarrow$ (iii): This follows from Proposition 2.13 because the semigroup $S_{z}(t):=$ $e^{t z Q}$ satisfies the conditions of Definition 4.1 for some angle in $(0, \pi / 2]$ if and only if $\sup _{\operatorname{Re} s>0}\left\|(1-s z Q)^{-1}\right\|<\infty$; see, e.g., [ABHN01, Corollary 3.7.12].

(iii) $\Rightarrow$ (i): Because $\mathcal{B}_{Q} \subset \mathcal{G}_{Q}$ by claim (iii) of Theorem 2.9, also their interiors satisfy $\mathcal{B}_{Q}^{\circ} \subset \mathcal{G}_{Q}^{\circ}$. Thus by Theorem 3.3 we get $\mathcal{R}_{Q} \backslash\{0\}=\mathcal{B}_{Q}^{\circ} \subset \mathcal{G}_{Q}^{\circ}$.

Proposition 4.4. A quasinilpotent $Q \in \mathcal{L}(X)$ satisfies $\mathcal{R}_{Q}=\mathcal{K}_{Q}^{\circ} \cup\{0\}$.

Proof. Let $s=r e^{i \phi} \in \mathbb{C}_{+}$and define $\theta$ by $s+1=|s+1| e^{i \theta}$. Then $|s+1|>|s|$, $\operatorname{Re} s>0$, and we obtain

$$
\frac{1}{|s+1|-|s|}<\frac{2|s+1|}{2 \operatorname{Re} s+1}<\frac{2|s+1|}{\operatorname{Re}(s+1)}=\frac{2}{\cos \theta} \leq \frac{2}{\cos \phi}
$$

where the last inequality holds because $0 \leq|\theta| \leq|\phi|<\pi / 2$ and thus $0<\cos \phi \leq$ $\cos \theta$. For $z \in \mathcal{K}_{Q}$ we thus have a constant $M_{z}<\infty$ such that

$$
\left\|(I-s z Q)^{-1}\right\| \leq \frac{M_{z}}{\cos \phi} \quad \text { for all } \quad s=r e^{i \phi} \in \mathbb{C}_{+} .
$$

If $z \in \mathcal{K}_{Q}^{\circ}$, we have a $\delta>0$ such that $e^{ \pm i \delta} z \in \mathcal{K}_{Q}$. Writing $s_{ \pm}:=s e^{ \pm i \delta}=|s| e^{i(\phi \pm \delta)}$ we see that there is a constant $M<\infty$ such that

$$
\left\|\left(I-s_{ \pm} z Q\right)^{-1}\right\| \leq \frac{M}{\cos \phi} \quad \text { for all } \quad s=r e^{i \phi} \in \mathbb{C}_{+} .
$$


Varying $\phi$ through the interval $(-\pi / 2+\delta / 2, \pi / 2-\delta / 2)$ (in which $1 / \cos \phi$ is bounded) proves that $\sup _{s^{\prime} \in \mathbb{C}_{+}}\left\|\left(I-s^{\prime} z Q\right)^{-1}\right\|<\infty$, and by Proposition 2.13 we have $z \in \mathcal{R}_{Q}$. The claim follows since $\mathcal{R}_{Q} \backslash\{0\}$ is open by Theorem 3.3.

Proposition 4.5. The set $\mathcal{K}_{Q}^{\infty}$ is convex for all quasinilpotent $Q \in \mathcal{L}(X)$.

Proof. We have $\mathcal{R}_{Q} \subset \mathcal{K}_{Q}^{\infty} \subset \mathcal{G}_{Q}$ by Theorem 2.9. If $\mathcal{R}_{Q}=\{0\}$, then $\mathcal{G}_{Q} \subset e^{i \phi} \overline{\mathbb{R}}_{+}$ for some $\phi \in[-\pi, \pi)$ by Theorem 4.3. Then there is nothing to prove because $\mathcal{K}_{Q}^{\infty}$ is star-like (hence, a convex subset of $e^{i \phi} \overline{\mathbb{R}}_{+}$) by Proposition 2.4.

If $\mathcal{R}_{Q} \neq\{0\}$, the sets $\mathcal{R}_{Q}$ and $\mathcal{G}_{Q}$ are sectors with the same interior $\mathcal{R}_{Q} \backslash\{0\}$. Thus, $\mathcal{K}_{Q}^{\infty}$ may fail to be convex only if the set $\mathcal{K}_{Q}^{\infty} \cap \partial \mathcal{G}_{Q}$ is not star-like which, however, is excluded by Proposition 2.4.

Theorem 4.6. For any quasinilpotent operator $Q \in \mathcal{L}(X)$ we have

$$
\mathcal{R}_{Q}=\mathcal{B}_{Q}^{\circ} \cup\{0\}=\mathcal{G}_{Q}^{\circ} \cup\{0\}=\mathcal{K}_{Q}^{\circ} \cup\{0\}=\left(\mathcal{K}_{Q}^{\infty}\right)^{\circ} \cup\{0\}
$$

If $\mathcal{R}_{Q} \neq\{0\}$, then $\overline{\mathcal{B}_{Q}}=\overline{\mathcal{G}_{Q}}=\overline{\mathcal{K}_{Q}^{\infty}}=\overline{\mathcal{R}_{Q}}$ and $\partial \mathcal{B}_{Q}=\partial \mathcal{G}_{Q}=\partial \mathcal{K}_{Q}^{\infty}=\partial \mathcal{R}_{Q}$.

Proof. The first equality is just a composition of claim (x) of Theorem 2.9 (giving first $\mathcal{B}_{Q}^{\circ} \subset\left(\mathcal{K}_{Q}^{\infty}\right)^{\circ} \subset \mathcal{G}_{Q}^{\circ}$ and hence the equality of interiors), Theorems $3.3,4.3$, and Proposition 4.4.

By what we have already proved, all of the sets $\mathcal{B}_{Q}, \mathcal{G}_{Q}, \mathcal{K}_{Q}^{\infty}$, and $\mathcal{R}_{Q}$ are convex with the same interior $\mathcal{R}_{Q} \backslash\{0\}$. To complete the proof, we show that for any convex set $K \subset \mathbb{C}$ with a nonempty interior $K^{\circ}$, the closures of $K^{\circ}$ and $K$ coincide.

Suppose that the inclusion $\overline{K^{\circ}} \subset \bar{K}$ of closures is strict, and choose $z \in \bar{K} \backslash \overline{K^{\circ}}$. Denote $\delta:=\operatorname{dist}\left(z, K^{\circ}\right)=\operatorname{dist}\left(z, \overline{K^{\circ}}\right)>0$. Because $z \in \bar{K}$, there exists a $z^{\prime} \in K$ such that $\left|z-z^{\prime}\right|<\delta / 3$, and thus $\operatorname{dist}\left(z^{\prime}, K^{\circ}\right)>\delta / 2$. Because $K$ is convex, it follows that

$$
\operatorname{conv}\left(z^{\prime}, D\right):=\left\{\alpha z^{\prime}+(1-\alpha) z: \alpha \in(0,1) \text { and } z \in D\right\} \subset K .
$$

where $D$ is any open disc of positive radius contained in $K^{\circ}$ (here we use the assumption that $K^{\circ}$ is nonempty). Since the set $\operatorname{conv}\left(z^{\prime}, D\right)$ is an open cone, it contains only interior points of $K$, and some of them are arbitrarily close to its vertex $z^{\prime}$. This is a contradiction against $\delta>0$.

There is an observation concerning the endpoints of $\mathcal{B}_{Q} \cap \partial \mathcal{B}_{Q}$ :

Proposition 4.7. We have $\mathcal{T}_{Q}^{1 / 2} \cap \mathcal{G}_{Q} \cap \partial \mathcal{G}_{Q} \subset \mathcal{B}_{Q} \cap \partial \mathcal{B}_{Q} \subset \overline{\mathcal{T}_{Q}^{1 / 2} \cap \mathcal{G}_{Q} \cap \partial \mathcal{G}_{Q}}$. One of the inclusions is strict if the set $\mathcal{B}_{Q} \cap \partial \mathcal{B}_{Q}$ is non-trivial (i.e., neither $\{0\}$, a full ray of infinite length, or a pair of such full rays).

Proof. If $\mathcal{R}_{Q} \neq\{0\}$ the first inclusion follows because $\mathcal{T}_{Q}^{1 / 2} \cap \mathcal{G}_{Q} \subset \mathcal{B}_{Q}$ by claim (v) of Theorem 2.9 and $\partial \mathcal{G}_{Q}=\partial \mathcal{B}_{Q}$ by Theorem 4.6. If $\mathcal{R}_{Q}=\{0\}$, use $\mathcal{G}_{Q} \cap \partial \mathcal{G}_{Q}=\mathcal{G}_{Q}$ and $\mathcal{B}_{Q} \cap \partial \mathcal{B}_{Q}=\mathcal{B}_{Q}$ instead.

For the second inclusion, we argue as follows: If $z \in \mathcal{B}_{Q} \cap \partial \mathcal{B}_{Q}$, we have for all $\alpha \in[0,1)$ the inclusion $\alpha x \in \mathcal{T}_{Q}^{1 / 2} \cap \mathcal{G}_{Q} \cap \partial \mathcal{G}_{Q}$ because $\alpha x \in \mathcal{B}_{Q} \subset \mathcal{G}_{Q}$ and $\alpha x \in \mathcal{T}_{Q}^{1 / 2}$ by claims (iii) and (viii) of Theorem 2.9 , and $\partial \mathcal{B}_{Q}=\partial \mathcal{G}_{Q}$ by Theorem 4.6 if $\mathcal{R}_{Q} \neq\{0\}$. If $\mathcal{R}_{Q}=\{0\}$, proceed as above. Letting $\alpha \rightarrow 1-$ proves that $x \in \overline{\mathcal{T}_{Q}^{1 / 2} \cap \mathcal{G}_{Q} \cap \partial \mathcal{G}_{Q}}$ in both cases. 
For contradiction, assume that at least one side of $\mathcal{B}_{Q} \cap \partial \mathcal{B}_{Q}$ is a closed ray of finite positive length, say $[0, z]$ for $z \in \mathbb{C}$. If $z \in \mathcal{T}_{Q}^{1 / 2}$, it follow from [Dun08b, Theorem 1.2] that $z / \beta \in \mathcal{B}_{Q} \cap \partial \mathcal{B}_{Q}$ for some $\beta \in(0,1)$ which is impossible.

Note that by [Dun08b, Theorem 1.2], claim (viii) of Theorem 2.9 cannot be improved to the inclusion $\mathcal{B}_{Q}=\mathcal{B}_{Q}[0,1] \subset \mathcal{T}_{Q}^{1 / 2}$ whenever $\mathcal{B}_{Q}$ does not consist of full rays.

\section{Growth on rays throught Tauberian sets}

We now give estimates on the growth of resolvents and semigoups on the rays that intersect the Tauberian sets $\mathcal{T}_{Q}$ or $\mathcal{T}_{Q}^{1 / 2}$.

Proposition 5.1. Let $Q \in \mathcal{L}(X)$ be a quasinilpotent operator and $z \in \mathbb{C}$.

(i) If $z \in \mathcal{T}_{Q}^{1 / 2}$, then there are constants $C_{r}, C_{g}<\infty$ (both depending on $z$ and given by (5.5)) such that

$$
\begin{aligned}
\left\|(1-s z Q)^{-1}\right\| & \leq C_{r}(s+1)^{1 / 2}+1 \quad \text { for all } \quad s \geq 0 ; \quad \text { and } \\
\left\|e^{t z Q}\right\| & \leq C_{g}\left(t^{1 / 2}+1\right) \quad \text { for all } t \geq 1 .
\end{aligned}
$$

(ii) If $z \in \mathcal{T}_{Q}$, then there are constants $D_{r}, D_{g}<\infty$ (both depending on $z$ and given by (5.8)) such that

$$
\begin{aligned}
\left\|(1-s z Q)^{-1}\right\| & \leq D_{r} \ln (s+1)+1 \quad \text { for all } \quad s>0 ; \quad \text { and } \\
\left\|e^{t z Q}\right\| & \leq D_{g}(\ln (t+1 / e)+4) \quad \text { for all } \quad t>1 .
\end{aligned}
$$

Proof. The proof is based on estimating the growth of powers of $T(z)=1+z Q$, $z \in \mathbb{C}$, using the identities

$$
\begin{aligned}
\left(1-\frac{\xi z}{1-\xi} Q\right)^{-1} & =(1-\xi)(I-\xi T(z))^{-1} \\
& =I-\sum_{j \geq 1} \xi^{j}(I-T(z)) T(z)^{j-1} \quad \text { for } \quad \xi \in \mathbb{D}, \\
T(z)^{k} & =I-\sum_{j=0}^{k-1}(I-T(z)) T(z)^{j}, \quad \text { and } \\
e^{t z Q} & =e^{-t} e^{t T(z)}=e^{-t} \sum_{j \geq 0} \frac{t^{j} T(z)^{j}}{j !} \quad \text { for } \quad t \in \mathbb{C}
\end{aligned}
$$

Claim (i): We prove the claims for $z \in \mathcal{T}_{Q}^{1 / 2}$ with the constants

$$
\begin{aligned}
C_{r} & :=\pi^{1 / 2} M \quad \text { and } \quad C_{g}:=(2 M+1) e^{1 / 2} \quad \text { where } \\
M & :=\sup _{j \geq 0}(j+1)^{1 / 2}\left\|(I-T(z)) T(z)^{j}\right\|<\infty .
\end{aligned}
$$

For all $\xi \in[0,1)$ we have

$$
\left\|\left(1-\frac{\xi z}{1-\xi} Q\right)^{-1}\right\| \leq 1+\frac{C_{r}}{\pi^{1 / 2}} \sum_{j \geq 1} \frac{\xi^{j}}{j^{1 / 2}}
$$


where we majorize the sum by an integral, noting that $\xi \in(0,1)$ and $-\infty<\ln \xi<0$ :

$$
\sum_{j \geq 1} \frac{\xi^{j}}{j^{1 / 2}} \leq \int_{0}^{\infty} \frac{\xi^{j} d j}{j^{1 / 2}}=\int_{0}^{\infty} \frac{\xi^{x^{2}} 2 x d x}{\left(x^{2}\right)^{1 / 2}}=\frac{1}{|\ln \xi|^{1 / 2}} \int_{-\infty}^{\infty} e^{-y^{2}} d y=\sqrt{\frac{\pi}{|\ln \xi|}} .
$$

Now for $s \in \mathbb{R}_{+}$we have $s=\frac{\xi}{1-\xi}$ if and only if $\xi=\frac{s}{s+1}$. Thus $|\ln \xi|=-\ln \xi=$ $\ln \frac{s+1}{s}=\ln (s+1)-\ln s \geq \frac{1}{s+1}$ by the mean value theorem, and thus (5.1) follows.

To estimate $\left\|e^{t z Q}\right\|$, we note that $\left\|T(z)^{j}\right\| \leq 1+2 M(j-1)^{1 / 2} \leq(2 M+1) j^{1 / 2}$ for $j \geq 1$, and we get

$$
\left\|e^{t z Q}\right\| \leq(2 M+1) e^{-t}\left(1+\sum_{j \geq 1} \frac{t^{j} \sqrt{j}}{j !}\right) \text { for } t \geq 0 .
$$

Assume that $t>1 / e$, and let $J$ be the integer satisfying $1 \leq J \leq e t<J+1$. Then

$$
\begin{aligned}
& \sum_{j \geq 1} \frac{t^{j} \sqrt{j}}{j !} \leq \sum_{j=1}^{J} \frac{t^{j} \sqrt{j}}{j !}+\sum_{j \geq J+1} \frac{t^{j} \sqrt{j}}{j !} \leq J^{1 / 2} e^{t}+\sum_{j \geq J+1} \frac{t^{j}}{(j-1) !} \\
& \leq J^{1 / 2} e^{t}+\frac{t^{J+1}}{J !}\left(1+\frac{t}{J+1}+\frac{t^{2}}{(J+1)(J+2)} \cdots\right) \\
& \leq J^{1 / 2} e^{t}+\frac{t^{J+1}}{J !} \sum_{k \geq 0}\left(\frac{t}{J+1}\right)^{k}=J^{1 / 2} e^{t}+\frac{t^{J+1}}{J !}\left(1-\frac{t}{J+1}\right)^{-1} .
\end{aligned}
$$

Since $J \geq 1$ we get

$$
\frac{t^{J+1}}{J !} \leq \frac{\left(\frac{J+1}{e}\right)^{J+1}}{\sqrt{2 \pi J}\left(\frac{J}{e}\right)^{J}} \leq \frac{1}{\sqrt{2 \pi J}}(J+1) \cdot e^{-1}\left(1+\frac{1}{J}\right)^{J} \leq \frac{1}{\sqrt{2 \pi}} \frac{J+1}{J^{1 / 2}}
$$

where we used Stirling's approximation $J !>\sqrt{2 \pi J}\left(\frac{J}{e}\right)^{J}$. Noting that et $-1<J \leq$ et, $\frac{t}{J+1}<e^{-1}$ and hence $\frac{1}{\sqrt{2 \pi}}\left(1-\frac{t}{J+1}\right)^{-1}<1$, we get

$$
\sum_{j \geq 1} \frac{t^{j} \sqrt{j}}{j !} \leq(e t)^{1 / 2} e^{t}+(e t+1)(e t-1)^{-1 / 2}
$$

Now (5.2) follows from (5.7) and $\max _{t \geq 1} e^{-t}\left(1+2(e t+1)(e t-1)^{-1 / 2}\right)<\sqrt{2}$.

Claim (ii): Take $z \in \mathcal{T}_{Q}$ and define the constants

$$
D_{r}:=\sup _{j \geq 0}(j+1)\left\|(I-T(z)) T(z)^{j}\right\|<\infty \quad \text { and } \quad D_{g}:=D_{r}+1 .
$$

The resolvent estimate of claim (ii) is proved as above but instead of equation (5.6) we now compute for $\xi \in[0,1)$ the sum

$$
\sum_{j \geq 1} \frac{\xi^{j}}{j}=\int_{0}^{\xi} \sum_{j \geq 1} x^{j-1} d x=\int_{0}^{\xi} \frac{d x}{1-x}=-\ln (1-\xi)=\ln (s+1) .
$$


It remains to estimate $\left\|e^{t z Q}\right\|$ when $z \in \mathcal{T}_{Q}$. Again, we have $\left\|T(z)^{j}\right\| \leq 1+$ $D_{r}(1+\ln j) \leq D_{g} \ln e j$ for $j \geq 1$, which gives the estimate just like (5.7)

$$
\left\|e^{t z Q}\right\| \leq D_{g} e^{-t}\left(1+\sum_{j \geq 1} \frac{t^{j} \ln (e j)}{j !}\right) \quad \text { for all } \quad t \geq 0 .
$$

Assume again that $t>1 / e$, and let $J$ be the integer satisfying $1 \leq J \leq e t<J+1$. Then

$$
\begin{aligned}
\sum_{j \geq 1} \frac{t^{j} \ln (e j)}{j !} & \leq \sum_{j=1}^{J} \frac{t^{j} \ln (e j)}{j !}+\sum_{j \geq J+1} \frac{t^{j} \ln (e j)}{j !} \\
& \leq e^{t} \ln (e J)+\frac{\ln e(J+1)}{J+1} \sum_{j \geq J+1} \frac{t^{j}}{(j-1) !} .
\end{aligned}
$$

The sum in the latter term can be estimated by Stirling's approximation together with the estimates et $<J+1 \leq e t+1, \frac{t}{J+1}<e^{-1}$, and $\frac{1}{\sqrt{2 \pi}}\left(1-\frac{t}{J+1}\right)^{-1}<1$ :

$$
\sum_{j \geq J+1} \frac{t^{j}}{(j-1) !} \leq \frac{t^{J+1}}{J !} \sum_{j \geq 0}\left(\frac{t}{J+1}\right)^{j}<J+1 .
$$

We get $\sum_{j \geq 1} \frac{t^{j} \ln (e j)}{j !} \leq e^{t} \ln (e J)+\ln e(J+1)<e^{t} \ln e(e t+1)$, and (5.4) follows since $\max _{t \geq 1} e^{-t} \ln e(e t+1)<1$.

\section{Consequences of the Phragmen-Lindelöf theorem}

Much of the next results are consequences of Phragmen-Lindelöf theorem (see Proposition 4.2) applied to the $\mathcal{L}(X)$-valued functions $r(s):=(1-s Q)^{-1}$ (the Fredholm resolvent) and $g(t):=e^{t Q}$. Because $\sigma(Q)=\{0\}$, the Gelfand formula for the spectral radius implies that for all $r>0$, there exists $C_{r}<\infty$ such that $\left\|e^{t Q}\right\| \leq C_{r} e^{r|t|}$ holds for all $t \in \mathbb{C}$; thus the entire function $g$ is always of exponential type. Unfortunately, the function $r$ does not have the same property without an additional compactness assumption on the quasinilpotent $Q$.

6.1. Conditions for $\mathcal{T}_{Q} \subset \mathcal{B}_{Q}$. The purpose of this section is to find sufficient conditions that imply $\mathcal{T}_{Q} \subset \mathcal{B}_{Q}$ for a quasinilpotent $Q$; i.e., that the Tauberian condition (1.4) implies the power-boundedness of $T(z)$. We already know that

$$
\begin{aligned}
& \mathcal{T}_{Q} \subset \mathcal{R}_{Q} \quad \Leftrightarrow \quad \mathcal{T}_{Q} \subset \mathcal{B}_{Q} \Leftrightarrow \\
& \mathcal{A}_{Q} \cap \mathcal{T}_{Q} \subset \mathcal{B}_{Q}, \quad \mathcal{T}_{Q} \cap\left(-\mathcal{G}_{Q}\right)=\emptyset, \quad \text { and } \quad \mathcal{T}_{Q} \cap\left(\mathcal{A}_{Q}^{c} \backslash\left(-\mathcal{G}_{Q}\right)\right)=\emptyset .
\end{aligned}
$$

The first equivalence holds by Proposition 3.5 because $\mathcal{R}_{Q}=\mathcal{B}_{Q}^{\circ} \cup\{0\}$. Based on claims (i) and (iv) of Theorem 2.9, we see that inclusion $\mathcal{A}_{Q} \cap \mathcal{T}_{Q} \subset \mathcal{B}_{Q}$ always holds. By Proposition 2.7, we have $\mathcal{T}_{Q} \cap\left(-\mathcal{G}_{Q}\right)=\emptyset$ for all $Q \neq 0$, too. Hence, we conclude that $\mathcal{T}_{Q} \subset \mathcal{B}_{Q}$ if and only if

$$
\mathcal{T}_{Q} \cap\left(\mathcal{A}_{Q}^{c} \backslash\left(-\mathcal{G}_{Q}\right)\right)=\emptyset
$$

and by a similar argument we see $\mathcal{T}_{Q}^{1 / 2} \subset \mathcal{A}_{Q}$ if and only if

$$
\mathcal{T}_{Q}^{1 / 2} \cap\left(\mathcal{A}_{Q}^{c} \backslash\left(-\mathcal{G}_{Q}\right)\right)=\emptyset \text {. }
$$


Clearly $(6.2) \Rightarrow(6.1)$, and we look for sufficient conditions to ensure either of these. Let us start from a simple observation when (6.2) holds trivially:

Proposition 6.1. Let $Q \in \mathcal{L}(X)$ be a quasinilpotent operator whose Ritt angle satisfies $\theta>\pi / 4$. Then $\mathcal{T}_{Q}^{1 / 2} \subset \mathcal{A}_{Q}$ and hence $\mathcal{T}_{Q} \subset \mathcal{B}_{Q}$.

Proof. Denote the Ritt angle of $Q$ by $\theta>0$. Then $\mathcal{A}_{Q}$ contains an open sector $\Sigma:=\mathbb{C}_{+} \mathcal{G}_{Q}$ whose central angle is $2 \theta+\pi$; see claim (ii) of Theorem 2.9. Because the central angle of the sector $-\mathcal{G}_{Q}$ is $2 \theta$ and $-\mathcal{G}_{Q}$ is geometrically opposite to $\Sigma$, we conclude that $\mathcal{A}_{Q} \cup\left(-\mathcal{G}_{Q}\right)$ contains open sectors whose total central angle is at least $\min (2 \pi, 4 \theta+\pi)$. If now $\theta>\pi / 4$, there is no room at all left for the set $\mathcal{A}_{Q}^{c} \backslash\left(-\mathcal{G}_{Q}\right)$, and (6.2) follows.

Proposition 6.2. If $Q \in \mathcal{L}(X)$ is a quasinilpotent operator such that the origin is an interior point of $\operatorname{conv}\left(\mathcal{G}_{Q} \cup \mathcal{T}_{Q}^{1 / 2}\right)$, then $Q=0$. If $\mathcal{R}_{Q} \neq\{0\}$ and $Q \neq 0$, then $\mathcal{A}_{Q} \not \subset \mathcal{T}_{Q}^{1 / 2}$.

Thus, the origin $z=0$ is a boundary point of all of the sets $\mathcal{B}_{Q}, \mathcal{G}_{Q}, \mathcal{K}_{Q}, \mathcal{K}_{Q}^{\infty}, \mathcal{R}_{Q}$, $\mathcal{T}_{Q}^{1 / 2}$, or $\sqcup_{Q}$ if and only if $Q \neq 0$.

Proof. If the origin is an interior point of $\operatorname{conv}\left(z_{1}, z_{2}, z_{3}\right)$ with $z_{1}, z_{2}, z_{3} \in \mathcal{G}_{Q} \cup \mathcal{T}_{Q}^{1 / 2}$, then the three rays $z_{1} \mathbb{R}_{+}, z_{2} \mathbb{R}_{+}$, and $z_{3} \mathbb{R}_{+}$divide $\mathbb{C}$ into three sectors whose central angles are strictly less than $\pi$. On these rays, the entire function $g(t)=e^{t Q}$ grows at most like a square root by claim (i) of Proposition 5.1. Since the entire function $g$ is of exponential type, Proposition 4.2 (with $\omega=1$ ) implies $\|g(t)\| \leq C\left(|t|^{1 / 2}+1\right)$ holds for some $C<\infty$ and all $t \in \mathbb{C}$. By the Cauchy estimates, such $g$ is a constant function and $Q=0$ follows.

Suppose $\mathcal{R}_{Q}=e^{i \phi} \Sigma_{\delta} \cup\{0\}$ for $\delta>0$ and $\phi \in[-\pi, \pi)$. By claim (ii) of Theorem 2.9 we have $\operatorname{conv}\left(\mathcal{A}_{Q}\right)=\mathbb{C}$. Now, if $\mathcal{A}_{Q} \subset \mathcal{T}_{Q}^{1 / 2}$ holds, then $Q=0$ follows from the first claim.

To get the main result of this section, we proceed to real operators on partially ordered complex function spaces. Let us assume that the Banach space $X$ is a complex function space, meaning that each $x \in X$ is actually a function $x: \Omega \rightarrow \mathbb{C}$ where $\Omega$ is a set of points. We say that $x \in X$ is real if $x(\omega) \in \mathbb{R}$ for all $\omega \in \Omega$, and positive if $x(\omega) \geq 0$ for all $\omega \in \Omega$. The conjugate and absolute value of $x$ are defined as usual by $\bar{x}(\omega):=\overline{x(\omega)}$ and $|x|(\omega):=|x(\omega)|$ for all $\omega \in \Omega$. We require from $X$ that

(i) $x \in X \Leftrightarrow \bar{x} \in X$ as well as $x \in X \Leftrightarrow|x| \in X$;

(ii) $\|\bar{x}\|=\|x \mid\|=\|x\|$ for all $x \in X$; and

(iii) $\|x\| \leq\|y\|$ if $0 \leq x(\omega) \leq y(\omega)$ for all $\omega \in \Omega$.

It follows from these properties that each $x \in X$ has a decomposition $x=x_{11}-$ $x_{12}+i\left(x_{21}-x_{22}\right)$ where all $x_{j, k} \in X$ are positive and satisfy $\left\|x_{j, k}\right\| \leq\|x\|$.

The conjugate of an operator $T \in \mathcal{L}(X)$ is defined by $\bar{T} x:=\overline{T \bar{x}}$ for $x \in X$, and the operator is called [positive] real if it maps [positive] real vectors in $X$ to [positive] real vectors. If $-T$ is positive, then $T$ is, of course, negative. All products of real operators are real, and the same holds for positive operators. Squares of negative operators are positive but the same does not generally hold for general real operators: e.g., $T^{2}=-I$ if $T=\left[\begin{array}{cc}0 & 1 \\ -1 & 0\end{array}\right]$. We always have $\overline{T x}=\bar{T} \bar{x}, \overline{\bar{T}}=T$ and 
$\|\bar{T}\|=\|T\|$ but $\|\bar{T} T\| \neq\|T\|^{2}$ for positive real $T=\left[\begin{array}{ll}0 & 1 \\ 0 & 0\end{array}\right]$. The operator conjugation is generally not an involution in $\mathcal{L}(X)$ since $\overline{T S}=\bar{T} \bar{S} \neq \bar{S} \bar{T}$ unless $[T, S]=0$. If $Q \in \mathcal{L}(X)$ is a quasinilpotent real operator, we have clearly $\overline{T(z)}=T(\bar{z}), \overline{T_{z}}=T_{\bar{z}}$, $\overline{e^{t z Q}}=e^{t \bar{z} Q}$ for $t \in \mathbb{R}$ which implies that all the microspectral sets $\mathcal{A}_{Q}, \mathcal{B}_{Q}, \mathcal{G}_{Q}$, $\mathcal{R}_{Q}, \mathcal{T}_{Q}$, and $\mathcal{T}_{Q}^{1 / 2}$ are conjugate symmetric. It is this symmetry that makes it possible to use Phragmen-Lindelöf theorem for excluding points in (6.1).

Theorem 6.3. Let $Q \in \mathcal{L}(X), Q \neq 0$, be a quasinilpotent operator such that $z Q$ is a positive real operator (in the sense described above) for some $z \in \mathbb{C}, z \neq 0$. If $\mathcal{G}_{Q} \neq\{0\}$, then $\mathcal{T}_{Q}=\mathcal{B}_{Q}^{\circ} \cup\{0\} \subset \mathcal{B}_{Q}$ and $\mathcal{T}_{Q}^{1 / 2} \subset \mathcal{A}_{Q}$.

The inclusion $\mathcal{T}_{Q}^{1 / 2} \subset \mathcal{A}_{Q}$ is strict if $\mathcal{R}_{Q} \neq\{0\}$ since $\operatorname{conv}\left(\mathcal{G}_{Q} \cup \mathcal{T}_{Q}^{1 / 2}\right) \subset e^{i \phi} \overline{\mathbb{C}}_{+}$for some half plane $e^{i \phi} \overline{\mathbb{C}}_{+} \subset \mathcal{A}_{Q}$; see Proposition 6.2. If $\mathcal{B}_{Q}=\mathcal{G}_{Q}=\mathcal{R}_{Q}=\{0\}$, then we only know (by the same proposition) that $\mathcal{T}_{Q}^{1 / 2}$ is contained in some closed half plane whose boundary contains the origin.

Proof. We may assume without loss of generality that $Q$ itself is a negative real operator (i.e., $z=-1$ in the statement of this theorem), and then all the sets $\mathcal{A}_{Q}$, $\mathcal{B}_{Q}, \mathcal{G}_{Q}, \mathcal{R}_{Q}, \mathcal{T}_{Q}$, and $\mathcal{T}_{Q}^{1 / 2}$ are conjugate symmetric (by the real operator property), and $\mathcal{G}_{Q} \subset \mathbb{C}_{+} \cup\{0\}$ (by negativity). We divide the proof into two cases depending whether $\mathcal{R}_{Q} \neq\{0\}$ or $\mathcal{R}_{Q}=\{0\}$ (but $\mathcal{G}_{Q} \neq\{0\}$ ). By claim (i) of Theorem 2.9, Theorem 3.3, and Proposition 3.2, to prove $\mathcal{T}_{Q}=\mathcal{B}_{Q}^{\circ} \cup\{0\}$ it is enough to show that $\mathcal{T}_{Q} \subset \mathcal{B}_{Q}$. By claims (i), (iv), and (v) of Theorem 2.9, it is enough to just show that $\mathcal{T}_{Q}^{1 / 2} \subset \mathcal{A}_{Q}$.

Case $\mathcal{R}_{Q} \neq\{0\}$ : Now $\mathcal{R}_{Q}=\Sigma_{\theta^{\prime}} \cup\{0\}$ for the Ritt angle $\theta^{\prime}>0$ and $\Sigma_{\pi / 2+\theta^{\prime}} \subset \mathcal{A}_{Q}$ (see claim (ii) of Theorem 2.9). We conclude from this by simple geometry that

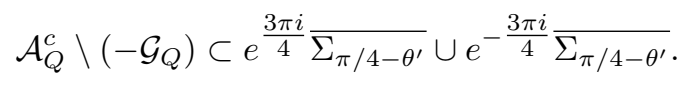

For contradiction, suppose that $\mathcal{T}_{Q}^{1 / 2} \subset \mathcal{A}_{Q}$ does not hold. Then by (6.1) and conjugate symmetry of $\mathcal{T}_{Q}^{1 / 2}$ there are points $r e^{i \phi}, r e^{-i \phi} \in \mathcal{T}_{Q}$ where $r>0$ and $\phi \in\left[\pi / 2+\theta^{\prime}, \pi-\theta^{\prime}\right]$. By claim (ii) of Proposition 5.1, the entire function $g(t)=e^{t Q}$ grows at most like a square root on the rays $e^{i \phi} \mathbb{R}_{+}$and $e^{-i \phi} \mathbb{R}_{+}$. Also $\overline{\mathbb{R}}_{+} \subset \mathcal{G}_{Q}$ by Theorem 4.3, and $g$ is bounded on this ray. These three rays divide the whole $\mathbb{C}$ into three sectors whose central angles are strictly less than $\pi$. Using the same argument as in the proof of Proposition 6.2, we conclude the contradiction $Q=0$. Thus $\mathcal{T}_{Q}^{1 / 2} \subset \mathcal{A}_{Q}$ follows as claimed.

Case $\mathcal{R}_{Q}=\{0\}$ but $\mathcal{G}_{Q} \neq\{0\}$ : Proposition 2.4 and Theorem 4.3 imply that $\mathcal{G}_{Q}=e^{i \phi} \overline{\mathbb{R}}_{+}$for some $\phi \in[-\pi, \pi)$. Hence $\mathcal{G}_{Q}=\overline{\mathbb{R}}_{+}$and $\mathbb{C}_{+} \subset \mathcal{A}_{Q}$ because $Q$ is negative real. Let us first prove that $\mathcal{T}_{Q}^{1 / 2} \subset \overline{\mathbb{C}}_{+}$.

For contradiction, suppose that $r e^{i \phi} \in \mathcal{T}_{Q}^{1 / 2}$ with $\phi \in(\pi / 2, \pi)$; note that $\phi=\pi$ is excluded by Proposition 2.7. By conjugate symmetry, also $r e^{-i \phi} \in \mathcal{T}_{Q}^{1 / 2}$. Now, the function $g(t)=e^{t Q}$ grows at most like a square root on the rays $e^{ \pm i \phi} \mathbb{R}_{+}$by claim (i) of Proposition 5.1. Since the function $g$ is bounded on $\mathbb{R}_{+} \subset \mathcal{G}_{Q}$, it follows that $Q=0$ just like in the first part of this proof. This contradiction proves $\mathcal{T}_{Q}^{1 / 2} \subset \overline{\mathbb{C}}_{+} \subset \overline{\mathcal{A}_{Q}}$. 
To conclude the proof, it remains to show that $\mathcal{T}_{Q}^{1 / 2} \cup i \mathbb{R}=\{0\}$. Suppose not, meaning that we should have $\pm i y \in \mathcal{T}_{Q}^{1 / 2}$ for $y>0$ by the conjugate symmetry of $\mathcal{T}_{Q}^{1 / 2}$. Clearly there exists $K^{\prime}>0$ such that $\left\|(1 \pm i y Q)^{k}\right\| \leq 1+K^{\prime} k^{1 / 2}$ for all $k \geq 0$, and thus $\left\|\left(1+y^{2} Q^{2}\right)^{k}\right\|=\left\|(1+i y Q)^{k}(1-i y Q)^{k}\right\| \leq 1+\left(2 K^{\prime}+1\right) k \leq K(k+1)$ for all $k \geq 0$ with $K:=2 K^{\prime}+1$.

Since $Q$ is a negative real operator, the operator $Q^{\prime}:=y^{2} Q^{2}$ is positive real. If $x \in X$ is a positive vector, then $\left|\left(1+e^{i \theta} Q^{\prime}\right)^{k} x\right|(\omega) \leq\left[\left(1+Q^{\prime}\right)^{k} x\right](\omega)$ for all $\omega \in \Omega$ and $\theta \in[-\pi, \pi)$, and thus $\left\|\left(1+e^{i \theta} Q^{\prime}\right)^{k} x\right\| \leq\left\|\left(1+Q^{\prime}\right)^{k} x\right\|$. Presenting any $x \in X$ in terms of four positive $x_{j, k} \in X$ satisfying $\left\|x_{j, k}\right\| \leq\|x\|$ for $j, k=1,2$, we obtain

$$
\left\|\left(1+e^{i \theta} Q^{\prime}\right)^{k}\right\| \leq 4\left\|\left(1+Q^{\prime}\right)^{k}\right\| \leq 4 K(k+1) \text { for all } \theta \in[\pi, \pi) \text { and } k \geq 0 .
$$

Using this estimate on power series of the exponential functions gives $\left\|e^{t\left(1+e^{i \theta} Q^{\prime}\right)}\right\| \leq$ $4 K(1+t) e^{t}$ for $t \geq 0$, and hence $\left\|e^{t Q^{\prime}}\right\| \leq 4 K(1+|t|)$ for all $t \in \mathbb{C}$. Since $\frac{1}{2 \pi i} \int e^{t Q^{\prime}} t^{-k+1} d t=Q^{\prime k} / k$ ! (where the integration is around any circle $r \mathbb{T}$ for $r>0)$, we obtain the estimate $\left\|Q^{\prime k}\right\| / k !=4 K(r+1) r^{-k+2}$ for all $r>0$. Putting $k=4$ and letting $r \rightarrow \infty$ gives $Q^{8}=0$. We conclude by Proposition 2.2 that $\mathcal{T}_{Q}^{1 / 2}=\{0\}$ which is a contradiction against $y>0$.

Even though we require from $X$ that its elements are functions defined on all of $\Omega$, it is not difficult to extend the definition of $X$ to spaces like $L^{p}(\Omega ; \mathbb{C}), 1 \leq p \leq \infty$ where $\Omega \subset R^{n}$ is equipped with the Lebesgue measure. Of course, then the partial ordering structure is defined only almost everywhere. This leads to Banach lattices but we leave such generalizations to the reader. Another way of doing this is to consider first the vector space $C(\Omega ; \mathbb{C})$ equipped with the $L^{p}$-norm and then proceeding by a density argument. With this extension, we see that the results of this section can be applied to Riemann-Liouville operators $V^{\alpha}$ (that are positive real) as introduced in Remark 2.3.

Proposition 6.4. Let $Q \in \mathcal{L}(X)$ be a quasinilpotent operator such that $z Q$ is a positive real operator for some $z \in \mathbb{C}, z \neq 0$. If $\mathcal{R}_{Q} \neq\{0\}$, then at least one of the following holds: (i) $\mathcal{T}_{Q}^{1 / 2} \subset \overline{\mathcal{G}_{Q}}$, or (ii) $\mathcal{B}_{Q}=\mathcal{G}_{Q}$.

By Corollary 2.12 we have either $\mathcal{T}_{Q}^{1 / 2} \subset \overline{\mathcal{G}_{Q}}$ or $\mathcal{G}_{Q} \subset \mathcal{T}_{Q}^{1 / 2}$ for such operators.

Proof. Without loss of generality assume that $Q$ is a negative real operator. Suppose that $\mathcal{R}_{Q} \neq\{0\}$ but $\mathcal{T}_{Q}^{1 / 2} \subset \overline{\mathcal{G}_{Q}}$ does not hold. Then there exists $z \in \mathbb{C}$ such that $z, \bar{z} \in \mathcal{T}_{Q}^{1 / 2} \backslash \overline{\mathcal{G}_{Q}}$. By interpolating between $z, \bar{z}$, and the points of the sector $\mathcal{R}_{Q}$ using Proposition 2.14, we conclude that $\partial \mathcal{G}_{Q} \subset \mathcal{T}_{Q}^{1 / 2}$; thus $\overline{\mathcal{G}_{Q}} \subset \mathcal{T}_{Q}^{1 / 2}$ since $\mathcal{G}_{Q}^{\circ}=\mathcal{B}_{Q}^{\circ} \subset \mathcal{T}_{Q} \subset \mathcal{T}_{Q}^{1 / 2}$ by Proposition 3.2 and Theorem 4.6. Now $\mathcal{B}_{Q}=\mathcal{G}_{Q}$ follows from claims (iii) and (v) of Theorem 2.9.

6.2. Quasinilpotent operators in Schatten classes. We proceed to compact quasinilpotent operators on a separable Hilbert space $X$. The approximation numbers are defined by $\sigma_{j}(Q):=\inf _{\text {rank } F=j}\|Q-F\|$ for $j=0,1, \ldots$ Clearly $\sigma_{0}(Q)=$ $\|Q\|$ and $\lim _{j \rightarrow \infty} \sigma_{j}(Q)=0$ is equivalent with the compactness of $Q$. For $p \in(0, \infty)$, the Schatten $p$-class $S_{p}(X)$ is defined by those $Q$ for which the norm

$$
\|Q\|_{S_{p}(X)}^{p}:=\sum_{j \geq 0} \sigma_{j}(Q)^{p}
$$


is finite. It is easy to see that $S_{p}(X) \subset S_{p^{\prime}}(X)$ for $p<p^{\prime}, S_{p}(X)$ is a Banach space under this norm, and it has also the ideal property $B Q \in S_{p}(X)$ whenever $B \in \mathcal{L}(X)$ and $Q \in S_{p}(X)$. For $p=1$ the space $S_{p}(X)$ is called the trace class, and we have the Hilbert-Schmidt operators for $p=2$. The Fredholm resolvent $r(s):=(1-s Q)^{-1}$ of quasinilpotent $Q$ is an entire function of finite exponential type $1 / p$ if $Q \in S_{p}(X)$. Indeed, we have the generalized Carleman inequality for $m \in \mathbb{N}$ and $p \in(m, m+1]$ :

$$
\left\|(I-s Q)^{-1}\right\| \leq m e^{3\|Q\|_{S_{p}(X)}|s|^{p}} \quad \text { for all } \quad s \in \mathbb{C} ;
$$

see, e.g., [Mal96, Theorem 5.4], [Nev93, Theorem 5.8.9], and [DS63, p. 1088-1119]. Using (6.3) makes it possible to apply Phragmen-Lindelöf theorem on Fredholm resolvent functions:

Proposition 6.5. Let $Q \in \mathcal{L}(X), Q \neq 0$, be a quasinilpotent operator such that $Q \in S_{p}(X)$ for some $p>1$. Then the Ritt angle of $Q$ satisfies $\theta \leq \frac{\pi}{2}(1-1 / p)$.

Proof. Denoting the Ritt angle by $\theta$, we conclude from claim (ii) of Theorem 2.9 that $\mathcal{A}_{Q}^{c}$ is contained in a closed sector $e^{i \phi} \overline{\Sigma_{\pi-2 \theta}}$ for some $\phi \in[-\pi, \pi)$. We thus have three rays in $\mathcal{A}_{Q}$ that divide $\mathbb{C}$ into three closed sectors $\Sigma_{i}, i=1,2,3$ so that the largest of their central angles $\alpha$ satisfies $\alpha=\pi-2 \theta+\epsilon$ where $\epsilon>0$ can be chosen arbitrarily small.

If $Q \in S_{p}(X)$ for $p>1$, then (6.3) holds and Proposition 4.2 can be applied to $(I-s Q)^{-1}$ on each $\Sigma_{i}$ separately. If $\alpha<\pi / p$, we conclude that $(I-s Q)^{-1}$ is bounded on all of $\mathbb{C}$, and the contradiction $Q=0$ follows from Liouville's theorem. Hence $\alpha \geq \pi / p$, and the proof is completed by letting $\epsilon \rightarrow 0+$.

Corollary 6.6. Let $Q \in \mathcal{L}(X), Q \neq 0$, be a quasinilpotent operator such that $Q \in S_{p}(X)$ for all $p>1$. Then $\mathcal{R}_{Q}=\{0\}$.

Considering the Riemann-Liouville operators $Q=-V^{\alpha}$ on $L^{2}(0,1)$ for $\alpha>0$ in Remark 2.3, their approximation number asymptotics are known to be $\sigma_{j}\left(V^{\alpha}\right) \approx$ $(\pi j)^{-\alpha}$; see [TG96]. Hence $V^{\alpha} \in S_{p}\left(L^{2}(0,1)\right)$ if and only if $p>1 / \alpha$. In particular, Corollary 6.6 implies $\mathcal{R}_{Q}=\{0\}$ for $Q=-V^{1}$, and the result is sharp by [Lyu01, p. 137].

By Theorem 3.3, the Ritt set cannot strictly contain any of the half planes $e^{i \phi} \mathbb{C}_{+} \cup\{0\}$ for $\phi \in[-\pi, \pi)$ if $Q \neq 0$. Even the restricting case is also impossible unless $(I-s Q)^{-1}$ is quite pathological:

Proposition 6.7. Let $Q \in \mathcal{L}(X)$ be a quasinilpotent operator such that $\mathcal{R}_{Q}=$ $e^{i \phi} \mathbb{C}_{+} \cup\{0\}$ for some $\phi \in[-\pi, \pi)$. Then the Fredholm resolvent $r(s)=(I-s Q)^{-1}$ is not an entire function of exponential type in the sense of (4.1). In particular, $Q \notin S_{p}(X)$ for all $p>0$.

Proof. If $Q$ is as assumed, then $\mathbb{C} \backslash e^{i \theta} \mathbb{R}_{+} \subset \mathcal{A}_{Q}$ for some $\theta \in[-\pi, \pi)$ by claim (ii) of Theorem 2.9. If $r$ is of some bounded type, we can divide the whole of $\mathbb{C}$ into a finite number of sufficiently small sectors and use Proposition 4.2 on each of them separately. Again, the boundedness of the entire function $r$ would follow, and hence $Q=0$. That $Q \notin S_{p}(X)$ follows from (6.3) for $p>1$, and from [Mal96, Theorem 3.8] for $p \in(0,1]$. 


\section{The Abel set $\mathcal{A}_{Q}$ Revisited}

Let us start by stating that if $\mathcal{R}_{Q} \neq\{0\}$, there are sectors inside $\mathcal{A}_{Q}$ on which the Fredholm resolvent $(I-s Q)^{-1}$ is uniformly bounded:

Lemma 7.1. Assume that $Q \in \mathcal{L}(X), Q \neq 0$, is a quasinilpotent operator such that $\mathcal{R}_{Q}$ has a nonempty interior. Define $E:=\mathcal{R}_{Q} \mathbb{C}_{+} \cap \mathbb{T}, E^{\prime \prime}:=\mathcal{A}_{Q} \cap \mathbb{T}$, and by $E^{\prime}$ denote the (path connected) component of $E^{\prime \prime}$ that contains $E$. Define the function $\Phi: E^{\prime \prime} \rightarrow \mathbb{R}_{+}$by setting

$$
\Phi\left(e^{i \theta}\right):=\sup _{r \geq 0}\left\|\left(I-r e^{i \theta} Q\right)^{-1}\right\| .
$$

Then $E$ and $E^{\prime}$ are circular intervals, $E \subset E^{\prime} \subset E^{\prime \prime}$, and the following holds:

(i) For any closed, circular interval $K \subset E$ we have $\sup _{e^{i \theta} \in K} \Phi\left(e^{i \theta}\right)<\infty$.

(ii) We have $\sup _{e^{i \theta} \in E} \Phi\left(e^{i \theta}\right)=\infty$.

(iii) For any closed, circular interval $K \subset E^{\prime}$, there is an open circular interval $W \subset K$ such that $\sup _{e^{i \theta} \in W} \Phi\left(e^{i \theta}\right)<\infty$.

Indeed, we do not know whether $\mathcal{A}_{Q} \backslash\{0\}$ is always a connected set. Proposition 7.3 shows that $E^{\prime}=E$ for $Q$ that satisfy a compactness assumption.

Proof. Claim (i): Because $t \mapsto e^{t Q}$ is an analytic semigroup on $\mathcal{R}_{Q}$ that is bounded on each closed subsector $\bar{\Sigma}$ of $\mathcal{R}_{Q}$, the Fredholm resolvent $(I-s Q)^{-1}$ is uniformly bounded on corresponding closed subsectors $\bar{\Sigma} \bar{C}_{+}$by the Hille-Yoshida theorem.

Claim (ii): If $\sup _{e^{i \theta} \in E} \Phi\left(e^{i \theta}\right)=\sup _{s \in \mathcal{R}_{Q} \mathbb{C}_{+}}\left\|(I-s Q)^{-1}\right\|<\infty$, we would conclude by Proposition 2.13 that the bounding ray(s) of $\mathcal{G}_{Q}$ in $\partial \mathcal{G}_{Q}$ would belong to $\mathcal{R}_{Q}$. This is impossible by Theorem 4.6 .

Claim (iii): The function $\Phi$ defined by (7.1) is clearly nonnegative and lower semicontinuous. Define the level sets for $k=1,2, \ldots$ by $E_{k}:=\left\{\theta \in E^{\prime}: \Phi\left(e^{i \theta}\right) \leq\right.$ $k\}$. By lower semicontinuity, all of these sets are closed, and clearly $E_{k} \subset E_{j}$ for $k<j$ as well as $E^{\prime}=\cup_{j \geq 1} E_{j}$. Defining $K_{j}:=K \cap E_{j}$ we get an increasing family of closed sets satisfying $K=\cup_{j \geq 1} K_{j}$. Because $K$ is a complete metric space, there exists $j \in \mathbb{N}$ such that $K_{j}$ has a non-empty open interior $K_{j}^{\circ}$ by Baire's category theorem. Hence, there exists an open circular interval $W \subset K_{j}^{\circ}$ such that $\Phi\left(e^{i \theta}\right) \leq j$ for all $e^{i \theta} \in W$. This completes the proof.

The operator $Q=-V^{\alpha}$ for $\alpha \in(0,1)$ provides us with an example of an operator for which $\mathcal{A}_{Q}$ is not convex:

Proposition 7.2. Let $Q \in \mathcal{L}(X)$ be quasinilpotent operator such that $\mathcal{R}_{Q} \neq\{0\}$. Then one of the following holds: (i) $\mathcal{A}_{Q}$ is not convex; (ii) the Fredholm resolvent $r(s):=(1-s Q)^{-1}$ is not of any exponential type; or (iii) $Q=0$.

Proof. Assume that $\mathcal{A}_{Q}$ is convex, and that $r$ is of some exponential type. We prove that $Q=0$.

Since $\mathcal{R}_{Q}$ has a nonempty interior, we see from claim (ii) of Theorem 2.9 that $\operatorname{conv}\left(\mathcal{A}_{Q}\right)=\mathbb{C}=\mathcal{A}_{Q}$. Thus the entire function $r$ is bounded on all rays in the sense that $\sup _{r>0}\left\|\left(I-r e^{i \theta} Q\right)^{-1}\right\|<\infty$ for all $\theta \in[-\pi, \pi)$. Proposition 4.2 implies that for any $e^{i \theta} \in \mathbb{T}$, there is an open sector $\Sigma(\theta)$ with $e^{i \theta} \in \Sigma(\theta)$ on which $r$ is uniformly bounded by some constant $C(\theta)<\infty$. The sets $\Sigma(\theta) \cap \mathbb{T}$ are an open cover for $\mathbb{T}$, and hence there is a finite sub-cover. From this it follows that $r$ is uniformly bounded on all of $\mathbb{C}$, and $Q=0$ by Liouville's theorem. 
We have $\mathcal{R}_{Q} \mathbb{C}_{+} \subset \mathcal{A}_{Q}$ by claim (ii) of Theorem 2.9 but we cannot exclude in Lemma 7.1 the possibility that $\mathcal{A}_{Q}$ could be substantially larger than $\mathcal{R}_{Q} \mathbb{C}_{+}$. If the Fredholm resolvent is of exponential type (e.g., if $Q \in S_{p}(X)$ for $p>0$ ), then we know that at least a part of $\partial \mathcal{A}_{Q}$ is where one would expect:

Proposition 7.3. Let $Q \in \mathcal{L}(X)$ be a quasinilpotent operator with $\mathcal{G}_{Q} \neq\{0\}$ and that the Fredholm resolvent $r(s)=(I-s Q)^{-1}$ is an entire function of exponential type. Then $\partial\left(\mathcal{G}_{Q} \mathbb{C}_{+}\right) \subset \partial \mathcal{A}_{Q}$.

Proof. We prove this under the stronger assumption $\mathcal{R}_{Q} \neq\{0\}$ in which case $\mathcal{G}_{Q} \mathbb{C}_{+}=\mathcal{R}_{Q} \mathbb{C}_{+}$holds because $\mathbb{C}_{+}$is open and $\mathcal{R}_{Q}=\mathcal{G}_{Q}^{\circ} \cup\{0\}$. Then, by claims (i) and (ii) of Lemma 7.1, the open sector $\mathcal{R}_{Q} \mathbb{C}_{+} \backslash\{0\}$ has the following properties: (i) for each closed sector $\Sigma \subset \mathcal{R}_{Q} \mathbb{C}_{+}$, the Fredholm resolvent $r$ is bounded on $\Sigma$, and (ii) there is no larger sector than $\mathcal{R}_{Q} \mathbb{C}_{+} \backslash\{0\}$ having the same property.

Since $\mathcal{R}_{Q}$ is a sector (see Theorem 3.3), we have $\partial\left(\mathcal{R}_{Q} \mathbb{C}_{+}\right)=e^{i \phi_{1}} \overline{\mathbb{R}_{+}} \cup e^{i \phi_{2}} \overline{\mathbb{R}_{+}}$

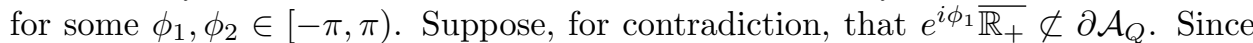
always $\mathcal{R}_{Q} \mathbb{C}_{+} \subset \mathcal{A}_{Q}$, it is impossible that $e^{i \phi_{1}} \mathbb{R}_{+} \subset \mathcal{A}_{Q}^{c} \backslash \partial \mathcal{A}_{Q}$. Hence, $e^{i \phi_{1}} \mathbb{R}_{+} \subset$ $\mathcal{A}_{Q}^{\circ}$ and we have $e^{i \phi_{1} \overline{\Sigma_{\theta}}} \subset \mathcal{A}_{Q}^{\circ} \cup\{0\} \subset \mathcal{A}_{Q}$ for all $\theta>0$ small enough. We may conclude that $r$ is bounded in $e^{i \phi_{1}} \overline{\Sigma_{\theta}}$ with sufficiently small $\theta>0$ that is compatible with the exponential type of $r$ so that Proposition 4.2 can be used. Now, the set $\Sigma^{\prime}:=e^{i \phi_{1}} \Sigma_{\theta} \cup \mathcal{R}_{Q} \mathbb{C}_{+}$is strictly larger than $\mathcal{R}_{Q} \mathbb{C}_{+} \backslash\{0\}$ but it still satisfies condition (i) given above.

\section{Miscellaneous observations}

We start by giving three results concerning the Ritt set $\mathcal{R}_{Q}$. We consider the case where $T_{z}$ in (1.3) is not only (uniformly) power-bounded as in Proposition 2.6 (related to Hille-Yoshida semigroup generator theorem) but more strongly, a Ritt operator characterized by the resolvent estimate

$$
\sup _{\xi \in 1+\Sigma_{\pi / 2+\delta}}|\xi-1| \cdot\left\|\left(\xi-T_{z}\right)^{-1}\right\|<\infty .
$$

For all $z \in \mathbb{C}$, define

$$
Q_{z}:=T_{z}-I=z Q(1-z Q)^{-1} .
$$

Proposition 8.1. Let $Q \in \mathcal{L}(X)$ be a quasinilpotent operator. Then $T_{z}$ in (1.3), (8.1) is a Ritt operator if and only if $z \in \mathcal{R}_{Q}$.

In other words, $z \in \mathcal{R}_{Q(I-z Q)^{-1}}$ if and only if $1 \in \mathcal{R}_{Q_{z}}$ if and only if $z \in \mathcal{R}_{Q}$. This does not mean that $\mathcal{R}_{Q}=\mathcal{R}_{Q(I-z Q)^{-1}}$ for all $z$.

Proof. For all $\xi \neq 1$ we have the identity

$$
(\xi-1)\left(\xi-T_{z}\right)^{-1}=(1-z Q)\left(1-\frac{\xi z}{\xi-1} Q\right)^{-1}
$$

Denoting $s=\xi /(\xi-1)$ it is easy to see that $\xi \in 1+\Sigma_{\pi / 2+\delta}$ is equivalent with $s \in 1+\Sigma_{\pi / 2+\delta}$. Since $\rho(Q)=0$ we have

$$
\sup _{\xi \in 1+\Sigma_{\pi / 2+\delta}}\left\|(\xi-1)\left(\xi-T_{z}\right)^{-1}\right\|<\infty \Leftrightarrow \sup _{s \in 1+\Sigma_{\pi / 2+\delta}}\left\|(1-s z Q)^{-1}\right\|<\infty
$$


for any $\delta>0$. Recall that $z \in \mathcal{R}_{Q}$ if and only if $\sup _{\operatorname{Re} s>-1 / 2}\left\|(1-s z Q)^{-1}\right\|<\infty$. Since the set $\{\operatorname{Re} s \geq-1 / 2\} \backslash\left(1+\Sigma_{\pi / 2+\delta}\right)$ is a closed triangle, and the mapping $s \mapsto(1-s z Q)^{-1}$ is continous for all $z$, we conclude that

$$
\sup _{s \in 1+\Sigma_{\pi / 2+\delta}}\left\|(1-s z Q)^{-1}\right\|<\infty \Leftrightarrow \sup _{\operatorname{Re} s>-1 / 2}\left\|(1-s z Q)^{-1}\right\|<\infty .
$$

This proves the claim.

It is clear that $\sup _{k \in \mathbb{N}}(k+1)\left\|\left(I-T_{z}\right) T_{z}^{k}\right\|<\infty$ for all $z \in \mathcal{R}_{Q}$ by [MNY09, Proposition 1.1] and Proposition 8.1. A similar but weaker conclusion can be given in the larger set $\mathcal{G}_{Q}$ :

Proposition 8.2. Let $Q \in \mathcal{L}(X)$ be a quasinilpotent operator. The operator $T_{z}$ in (1.3) satisfies $\sup _{k \in \mathbb{N}} \sqrt{k+1}\left\|\left(I-T_{z}\right) T_{z}^{k}\right\|<\infty$ for all $z \in \mathcal{G}_{Q}$.

Proof. Let $z \in \mathcal{G}_{Q}$ be arbitrary. Define for $\alpha \in(0,1)$ the power-bounded operators $R_{\alpha, z}:=(1-\alpha)+\alpha T_{z}=I+\alpha Q_{z}$; see Proposition 2.6 above. Since $I-R_{\alpha, z}=$ $-\alpha z Q(1-z Q)^{-1}$, we get by a straightforward computation

$$
-\frac{1}{\alpha}\left(I-R_{\alpha, z}\right) R_{\alpha, z}^{k} \cdot(1-(1-\alpha) z Q)^{-k}=z Q(1-z Q)^{-k-1} .
$$

The Hille-Yosida theorem implies that $\sup _{k \in \mathbb{N}}\left\|(1-(1-\alpha) z Q)^{-k}\right\|<\infty$ because $1-\alpha>0$ and $z \in \mathcal{G}_{Q}$. Moreover, it follows from [Nev93, Theorem 4.5.3] that

$$
\sup _{k \in \mathbb{N}} \sqrt{k+1}\left\|\left(I-R_{\alpha, z}\right) R_{\alpha, z}^{k}\right\|<\infty .
$$

Noting that $z Q(1-z Q)^{-k-1}=\left(I-T_{z}\right) T_{z}^{k}$, the claim follows from (8.2).

Note that $T_{z}=1+z Q+z^{2} Q^{2}(1-z Q)^{-1}$ approximates the operator $T(z)=$ $1+z Q$ for $z \approx 0$. If, instead of Proposition 8.2, we had $\mathcal{G}_{Q} \subset \mathcal{T}_{Q}^{1 / 2}$, then the equality $\mathcal{B}_{Q}=\mathcal{G}_{Q}$ would follow by Corollary 2.12. This is the motivation for Propositions 8.1 and 8.2 .

We complete this section by showing that the boundedness of the Fredholm resolvent in small sectors implies practically nothing on the the semigroups:

Proposition 8.3. There exists a quasinilpotent operator $Q \in \mathcal{L}(X)$ such that $\sup _{s \in \Sigma_{\delta}}\left\|(I-s Q)^{-1}\right\|<\infty$ with some $0<\delta<\pi / 2$ (hence, $\Sigma_{\delta} \subset \mathcal{A}_{Q}$ ) but the estimate

$$
\left\|e^{t Q}\right\| \leq M_{k} t^{k} \text { for all } t \geq 0
$$

does not hold for any $k \geq 1$ and $M_{k}<\infty$.

The operator $Q=-V^{1}$ in Remark 2.3 is sectorial so that $\sup _{s \in \Sigma_{\pi / 2-\eta}}\left\|(I-s Q)^{-1}\right\|<$ $\infty$ for all $\eta \in(0, \pi / 2)$; see, e.g., [Lyu01].

Proof. By $Q^{\prime}$ denote any quasinilpotent operator with $\mathcal{R}_{Q^{\prime}} \neq\{0\}$ (see Remark 2.3). Such $Q^{\prime}$ is never nilpotent by Proposition 2.2, and without loss of generality we may assume that $\mathcal{R}_{Q^{\prime}}=\Sigma_{2 \delta} \cup\{0\}$ for $\delta>0$. Then by claim (ii) of Theorem 2.9 we have $e^{ \pm i(\pi / 2+\delta)} \Sigma_{\delta} \subset \mathcal{A}_{Q^{\prime}}$, and also $\sup _{s^{\prime} \in e^{ \pm i(\pi / 2+\delta)} \Sigma_{\delta}}\left\|\left(I-s Q^{\prime}\right)^{-1}\right\|<\infty$ as can be seen by Proposition 2.8. Both the operators $Q^{ \pm}:=e^{ \pm i(\pi / 2+\delta)} Q^{\prime}$ satisfy now the conditions of this proposition.

For contradiction, suppose that $\left\|e^{t Q^{ \pm}}\right\| \leq M_{k} t^{k}$ for some $k, M_{k}$ and all $t \geq 0$. Then we would have $\left\|e^{t Q^{\prime}}\right\| \leq M_{k}|t|^{k}$ on the rays $e^{ \pm i(\pi / 2+\delta)} \mathbb{R}_{+}$, and also $\left\|e^{t Q^{\prime}}\right\| \leq$ 
$M<\infty$ on $\mathbb{R}_{+}$. Proposition 6.4 implies that $\left\|e^{t Q^{\prime}}\right\| \leq M_{k}|t|^{k}$ for all $t \in \mathbb{C}$, and hence $e^{t Q^{\prime}}$ is a polynomial. This is possible only if $Q$ is nilpotent which is impossible by Proposition 2.2.

\section{Conclusions}

These conclusions concern the open problems that remain.

9.1. Do the sets $\mathcal{B}_{Q}$ and $\mathcal{K}_{Q}^{\infty}$ consist of full rays? In other words, do we have $\mathcal{B}_{Q} \mathbb{R}_{+}=\mathcal{B}_{Q}$ ? We know that $\mathcal{B}_{Q}$ is convex. Since the open interior $\mathcal{B}_{Q}^{\circ}$ is a sector, it has the full ray property. Thus, the question can be rephrased whether $\left(\partial \mathcal{B}_{Q} \cap \mathcal{B}_{Q}\right) \mathbb{R}_{+} \subset \mathcal{B}_{Q}$. The same questions can be asked about the set $\mathcal{K}_{Q}^{\infty}$, too.

9.2. Do we always have $\mathcal{B}_{Q}=\mathcal{G}_{Q}$ ? If this equality holds, then Problem 9.1 is clearly resolved in positive. Since the convex sets $\mathcal{B}_{Q}$ and $\mathcal{G}_{Q}$ have the same interior, the question is whether $\partial \mathcal{B}_{Q} \cap \mathcal{B}_{Q}=\partial \mathcal{G}_{Q} \cap \mathcal{G}_{Q}$. See Corollary 2.12.

9.3. Is it possible to have $\mathcal{B}_{Q} \neq \mathcal{R}_{Q}$ or even $\mathcal{G}_{Q} \neq \mathcal{R}_{Q}$ when $\mathcal{R}_{Q} \neq\{0\}$ ? In other words, is one of the sets $\partial \mathcal{B}_{Q} \cap \mathcal{B}_{Q}$ and $\partial \mathcal{G}_{Q} \cap \mathcal{G}_{Q}$, or both, always empty for such $Q$ ? If they are, then Problem 9.2 is resolved in positive for operators with $\mathcal{R}_{Q} \neq\{0\}$.

9.4. Does the set $\mathcal{K}_{Q}$ consist of full rays, or is it convex? It would follow from convexity that Theorem 4.6 could be completed with $\overline{\mathcal{R}_{Q}}=\overline{\mathcal{K}_{Q}}$.

9.5. Do we always have $\mathcal{G}_{Q} \cap \partial \mathcal{T}_{Q} \subset \mathcal{T}_{Q}^{1 / 2}$ ? This inclusion has a flavour of a trace theorem, and there is certain ring of truth in it. If $\partial \mathcal{T}_{Q} \subset \mathcal{T}_{Q}^{1 / 2}$ holds for some $Q$ with $\mathcal{R}_{Q} \neq\{0\}$, then $\partial \mathcal{G}_{Q} \subset \mathcal{T}_{Q}^{1 / 2}$ since $\partial \mathcal{G}_{Q}=\partial \mathcal{B}_{Q}=\partial \mathcal{B}_{Q}^{\circ}$ and $\mathcal{A}_{Q} \cap \mathcal{T}_{Q}=\mathcal{R}_{Q}=\partial \mathcal{B}_{Q}^{\circ} \cup\{0\}$. This would again resolve Problem 9.2 in positive for operators with $\mathcal{R}_{Q} \neq\{0\}$.

9.6. Do we have the inclusion $\mathcal{T}_{Q} \subset \mathcal{B}_{Q}$ or even $\mathcal{T}_{Q}^{1 / 2} \subset \mathcal{A}_{Q}$ without the extra assumptions of Section 6? This is a particularly deep question, and Theorem 6.3 gives hints what kind of counter examples could work. Note that there is a counter example [KMSOT04, Theorem 3.3] for the same question without the quasinilpotency assumption.

All of these open problems seem to be resistent to the techniques we have presented in this work. We probably need fresh, new ideas now, and trying to produce counter examples seems like a reasonable next step.

\section{REFERENCES}

[ABHN01] W. Arendt, C. Batty, M. Hieber, and R. Neubrander, Vector-valued Laplace transforms and Cauchy problems, Birkhäuser, 2001.

[Ber83] M. Berkani, Inégalités et propriétés spectrales dans les algébres de Banach, Thèse de 3éme cycle, University of Bordeaux I, 1983.

[DS63] N. Dunford and J. Schwartz, Linear operators; part ii: Spectral theory, Interscience Publishers, Inc. (J. Wiley \& Sons), New York, 1963.

[Dun08a] N. Dungey, On an integral of fractional power operators, Colloquium Mathematicum 117 (2008), no. 2, 157-164.

[Dun08b] On time regularity and related conditions for power bounded operators, Proc. London Math. Soc. 97 (2008), no. 1, 97-116. 
[Est83] J. Esterle, Quasimultipliers, representations of $H^{\infty}$, and the closed ideal problem for commutative Banach algebras, Radical Banach algebras and automatic continuity (Long Beach, Calif., 1981) (Berlin), Lecture Notes in Mathematics, vol. 975, Springer Verlag, 1983, pp. 66-162.

[FNRR90] C. K. Fong, E. A. Nordgren, H. Radjavi, and P. Rosenthal, Weak resolvents of linear operators, II, Indiana University Mathematics Journal 39 (1990), no. 1, 67-83.

[FW73] S. R. Foguel and B. Weiss, On convex power series of a conservative Markov operator, Proceedings of the american mathematical society 38 (1973), no. 2, 325-330.

[KMSOT04] N. Kalton, S. Montgomery-Smith, K. Oleszkiewicz, and Y. Tomilov, Power-bounded operators and related norm estimates, J. London Math. Soc. (2) 70 (2004), no. 2, 463-478. MR MR2078905 (2005e:47020)

[Lyu01] Yu. Lyubich, The single-point spectrum operators satisfying Ritt's resolvent condition, Studia Mathematica 145 (2001), 135-142.

[Lyu10] . The power boundedness and resolvent conditions for functions of the classical Volterra operator, Studia Mathematica 196 (2010), no. 1, 41-63.

[Mal96] J. Malinen, On the properties for iteration of a compact operator with unstructured perturbation, Tech. Report A360, Helsinki University of Technology Institute of Mathematics, 1996.

[MNTY07] J. Malinen, O. Nevanlinna, V. Turunen, and Z. Yuan, A lower bound for the differences of powers of linear operators, Acta Mathematica Sinica 23 (2007), no. 4, $745-748$.

[MNY09] J. Malinen, O. Nevanlinna, and Z. Yuan, On the Tauberian condition for bounded linear operators, Mathematical Proceedings of the Royal Irish Academy 109 (2009), 101-108.

[Nev93] O. Nevanlinna, Convergence of iterations for linear equations, Lectures in Mathematics ETH Zürich, Birkhäuser Verlag, Basel, Boston, Berlin, 1993.

[Nev97] O. Nevanlinna, On the growth of the resolvent operators for power bounded operators, Linear Operators (F.H. Szafraniec J. Janas and J. Zemánek, eds.), Banach Center Publications, vol. 38, Inst. Math., Polish Acad. Sci., 1997, pp. 247-264.

[NRR87] E. A. Nordgren, H. Radjavi, and P. Rosenthal, Weak resolvents of linear operators, Indiana University Mathematics Journal 36 (1987), no. 4, 913-934.

[Pey69] A. Peyerimhoff, Lectures on summability, vol. 107, Springer Verlag, Berlin, 1969.

[TG96] V. K. Tuan and R. Gorenflo, Asymptotics of singular values of Volterra integral operators, Numerical Functional Analysis and Applications 17 (1996), 453-461.

[Ton89] Y.S. Tong, Quasinilpotent integral operators, Acta Mathematica Sinica 32 (1989), $727-735$.

[Tse03] D. Tsedenbayar, On the power boundedness of certain Volterra operator pencils, Studia Mathematica 156 (2003), no. 1, 59-66.

[Whi87] R. Whitley, The spectrum of a Volterra composition operator, Integral Equations Operator Theory 10 (1987), 146-149.

[Zem94] J. Zemánek, On the Gelfand-Hille theorems, Functional Analysis and Operator Theory (J. Zemánek, ed.), vol. 30, Banach center publications, 1994, pp. 369-385.

Jarmo Malinen, Department of Mathematics and Systems Analysis, Aalto University, P.O.Box 11100, 00076 AALTO, Finland. Jarmo.Malinen@aalto.fi. 